\title{
New Coumarins from Clausena lansium Twigs
}

\author{
Wisanu Maneerat, ${ }^{a}$ Uma Prawat, ${ }^{b}$ Nisakorn Saewan ${ }^{c}$ and Surat Laphookhieo*,a \\ ${ }^{a}$ Natural Products Research Laboratory, School of Science, Mae Fah Luang University, Tasud, \\ Muang, 57100 Chiang Rai, Thailand \\ ${ }^{b}$ Faculty of Science and Technology, Phuket Rajabhat University, Muang, 83000 Phuket, Thailand \\ ${ }^{c}$ School of Cosmetic Science, Mae Fah Luang University, Tasud, Muang, \\ 57100 Chiang Rai, Thailand
}

\begin{abstract}
Duas novas cumarinas, Clausenalansimin A (5) e B (9), juntamente com sete cumarinas conhecidas (1-4 e 6-8), foram isoladas de galhos de Clausena lansium. Todos os compostos foram determinados por métodos espectroscópicos. Alguns dos compostos isolados apresentaram citotoxicidade contra linhagens de células humanas cancerígenas (KB, MCF7 e NCI-H187).

Two new coumarins namely Clausenalansimin A (5) and B (9) together with seven known coumarins (1-4 and 6-8), were isolated from twigs of Clausena lansium. All compounds were determined by spectroscopic methods. Some of isolates had cytotoxicity against human cancer cell lines (KB, MCF7 and NCI-H187).
\end{abstract}

Keywords: Clausena lansium, coumarin, clausenalansimin A, clausenalansimin B, cytotoxicity

\section{Introduction}

Clausena lansium (Lour.) Skeels is a distant relative of citrus fruit belonging to the Rutaceae family. Several parts of this plant have been used as a folk medicine in China and Taiwan. For example, the leaves have been used for the treatment of coughs, asthma and gastro-intestinal diseases, and the seeds for acute and chronic gastrointestinal inflammation and ulcers. ${ }^{1}$ In addition, the fruits are used for influenza, colds and abdominal colic pains in the Philippines. ${ }^{2}$ Recently, the seed extract of $C$. lansium was found to exhibit antifungal, antiproliferative, and HIV reverse transcriptase-inhibitory activities. ${ }^{3}$ Previous chemical investigations of this plant have revealed a number of alkaloids and coumarins..$^{2,4-6}$ As parts of our continuing study on chemical constituents and biological activity of Thai medicinal plants, we report herein the isolation and structure elucidation of two new coumarins (5 and 9) along with seven known coumarins $(\mathbf{1 - 4}, \mathbf{6 - 8})$ from the twigs of C. lansium as well as the evaluation of cytotoxicity against KB, MCF7 and NCI-H187 cancer cell lines. In addition, the ${ }^{1} \mathrm{H}$ and ${ }^{13} \mathrm{C}$ NMR spectral data of $\mathbf{7}$ are also reported herein for the first time.

\footnotetext{
*e-mail: surat@mfu.ac.th; laphookhieo@yahoo.com
}

\section{Results and Discussion}

The combination of $\mathrm{CH}_{2} \mathrm{Cl}_{2}$ and acetone extracts of twigs of $C$. lansium was separated by chromatographic techniques to yield two new coumarins, clausenalansimin A (5) and B (9), together with seven known compounds (1-4, 6-8). All structures were elucidated using spectroscopic data and compared with those reported in the literature.

Clausenalansimin A (5) was isolated as yellow viscous oil. Its molecular formula was established as $\mathrm{C}_{21} \mathrm{H}_{22} \mathrm{O}_{5}$ by HRMS. The UV-Vis spectrum showed an absorption band of a conjugated furanocoumarin at 204-300 $\mathrm{nm},{ }^{7}$ whereas IR spectroscopic data displayed absorption bands of hydroxyl and carbonyl functionalities at 3442 and $1722 \mathrm{~cm}^{-1}$, respectively. The ${ }^{1} \mathrm{H}$ NMR spectrum (Table 1) of $\mathbf{5}$ showed the common signals of furanocoumarin similar to that of $\mathbf{1}$ at $\delta 6.36$ (1H, d, J 9.6 Hz, H-3), 7.36 (1H, d, J $\left.2.0 \mathrm{~Hz}, \mathrm{H}-2^{\prime}\right)$, $6.81(1 \mathrm{H}, \mathrm{s}, \mathrm{H}-5), 7.68\left(1 \mathrm{H}, \mathrm{d}, J 2.0 \mathrm{~Hz}, \mathrm{H}-3^{\prime}\right)$ and 7.76 $(1 \mathrm{H}, \mathrm{d}, J 9.6 \mathrm{~Hz}, \mathrm{H}-4) .{ }^{8}$ The main different signals were observed at $5.68\left(1 \mathrm{H}, \mathrm{dt}, J 7.2\right.$ and $\left.1.2 \mathrm{~Hz}, \mathrm{H}-2^{\prime \prime}\right), 5.11$ (1H, m, H-6"), 5.04 (2H, d, J $\left.7.2 \mathrm{~Hz}, \mathrm{H}-1^{\prime \prime}\right), 4.41$ (1H, m, H-5"), 2.17 (2H, m, H-4"), 1.76 (3H, s, H-9"), $1.68\left(3 \mathrm{H}, \mathrm{s}, \mathrm{H}-8^{\prime \prime}\right) 1.65$ (3H, s, H-10") in the ${ }^{1} \mathrm{H}$ NMR spectral data and could be identified as the 5-hydroxy- 
3,7-dimethylocta-2,7-dienyloxy moiety. This moiety was also confirmed by COSY and HMBC correlations (Figure 2 ) and located at $\mathrm{C}-8$ because the $\mathrm{H}-1^{\prime \prime}(\delta 5.04)$ showed ${ }^{3} J$ HMBC correlation with the carbon at C-8 $(\delta$ 131.5). Therefore, clausenalansimin A was deduced to be $\mathbf{5}$.

Clausenalansimin B (9) was isolated as yellow viscous oil with a molecular formula of $\mathrm{C}_{19} \mathrm{H}_{18} \mathrm{O}_{6}$ on the basis of HREIMS. The ${ }^{1} \mathrm{H}$ NMR spectral data of 9 revealed an $\alpha, \beta$-unsaturated lactone at $\delta 6.12(1 \mathrm{H}, \mathrm{d}, J 9.6 \mathrm{~Hz}, \mathrm{H}-3)$ and $7.96(1 \mathrm{H}, \mathrm{d}, J 9.6 \mathrm{~Hz}, \mathrm{H}-4)$ and two meta-coupling aromatic protons at $\delta 6.48(1 \mathrm{H}, \mathrm{d}, J 1.6 \mathrm{~Hz}, \mathrm{H}-6)$ and $6.26(1 \mathrm{H}, \mathrm{d}, J 1.6 \mathrm{~Hz}, \mathrm{H}-8) .{ }^{9}$ These results implied that this molecule is a 5,7-oxygenated coumarin nucleus. Moreover, the ${ }^{1} \mathrm{H}$ NMR spectrum also showed signals of $-\mathrm{OCH}_{2}-\mathrm{CH}=\mathrm{C}-$ unit at $\delta 4.66\left(2 \mathrm{H}, \mathrm{m}, \mathrm{H}-1^{\prime \prime}\right)$ and 5.53 $\left(1 \mathrm{H}, \mathrm{t}, J 5.6 \mathrm{~Hz}, \mathrm{H}-2^{\prime \prime}\right)$ and an $\alpha, \beta$-unsaturated $\gamma$-lactone moiety at $\delta 2.35\left(1 \mathrm{H}, \mathrm{dd}, J 17.0,7.2 \mathrm{~Hz}, \mathrm{H}-4 \mathrm{a}^{\prime \prime}\right), 2.61$ $\left(1 \mathrm{H}, \mathrm{dd}, J\right.$ 17.0, $\left.5.2 \mathrm{~Hz}, \mathrm{H}-4 \mathrm{~b}^{\prime \prime}\right), 5.11\left(1 \mathrm{H}, \mathrm{m}, \mathrm{H}-5^{\prime \prime}\right)$, 7.09 ( $1 \mathrm{H}$, br t, H-6"), 1.92 (3H, br s, H- $\left.8^{\prime \prime}\right) .{ }^{10}$ The side chain unit was also supported by COSY and HMBC experiments (Figure 2) and located at C-5 due to the ${ }^{2} J$ and ${ }^{3} J$ HMBC correlations of the H-4 $(\delta$ 7.96), H-6 ( $\delta$ $6.48)$ and $\mathrm{H}-1^{\prime \prime}(\delta 4.66)$ with $\mathrm{C}-5(\delta 156.1)$. The structure of clausenalansimin B, therefore, was assigned to 9 .

The remaining seven known coumarins included xanthotoxol (1), ${ }^{7}$ imperatorin $(\mathbf{2}),{ }^{11}$ heraclenin $(\mathbf{3}),{ }^{12}$ heraclenol (4), ${ }^{13}$ wampetin (6), ${ }^{14}$ indicolactonediol (7), ${ }^{15}$ and isoscopoletin $(\mathbf{8})^{16}$ were determined by the 1D and 2D NMR spectral data and comparison with their reported physical and spectroscopic data. In addition, the complete assignments of ${ }^{1} \mathrm{H}$ and ${ }^{13} \mathrm{C}$ NMR of indicolactonediol (7) are also reported herein for the first time (Table 1).

Only the stable compounds and sufficient quantity were evaluated for their cytotoxicity against three human cancer cell lines including oral cavity cancer (KB), breast cancer (MCF7) and small cell lung cancer (NCI-H187). The results of cytotoxicity of the tested compounds (1-3, 5 and $\mathbf{6}$ ) are summarized in Table 2. All these compounds showed weak activity with cytotoxicity against KB, MCF7 and NCI-H187 cell lines, except coumarins 3 and 6 which were found to be inactive with MCF7 cancer cell line.

Table 1. ${ }^{1} \mathrm{H}$ and ${ }^{13} \mathrm{C}$ NMR spectral data of $\mathbf{5 , 7}$ and 9 in $\mathrm{CDCl}_{3}$

\begin{tabular}{|c|c|c|c|c|c|c|}
\hline \multirow[t]{2}{*}{ Position } & \multicolumn{2}{|c|}{5} & \multicolumn{2}{|c|}{7} & \multicolumn{2}{|c|}{9} \\
\hline & $\delta_{\mathrm{H}}(J$ in $\mathrm{Hz})$ & $\delta_{\mathrm{C}}$ & $\delta_{\mathrm{H}}(J$ in $\mathrm{Hz})$ & $\delta_{\mathrm{C}}$ & $\delta_{\mathrm{H}}(J$ in $\mathrm{Hz})$ & $\delta_{\mathrm{C}}$ \\
\hline 1 & - & - & - & - & - & - \\
\hline 2 & - & 160.4 & - & 160.4 & - & 162.1 \\
\hline 3 & $6.36(\mathrm{~d}, 9.6)$ & 114.7 & $6.37(\mathrm{~d}, 9.6)$ & 114.8 & $6.12(\mathrm{~d}, 9.6)$ & 110.3 \\
\hline 4 & $7.76(\mathrm{~d}, 9.6)$ & 144.3 & $7.78(\mathrm{~d}, 9.6)$ & 144.3 & $7.96(\mathrm{~d}, 9.6)$ & 139.2 \\
\hline $4 a$ & - & 116.5 & - & 116.5 & - & 103.8 \\
\hline 5 & $7.36(\mathrm{~s})$ & 113.4 & $7.42(\mathrm{~s})$ & 114.0 & - & 156.1 \\
\hline 6 & - & 125.8 & - & 126.0 & $6.48(\mathrm{~d}, 1.6)$ & 96.4 \\
\hline 7 & - & 148.6 & - & 148.3 & - & 161.0 \\
\hline 8 & - & 131.5 & - & 131.4 & $6.26(\mathrm{~d}, 1.6)$ & 96.9 \\
\hline $8 \mathrm{a}$ & - & 143.8 & - & 143.5 & - & 156.6 \\
\hline $2^{\prime}$ & $7.68(\mathrm{~d}, 2.0)$ & 146.7 & $7.71(\mathrm{~d}, 2.0)$ & 146.8 & - & - \\
\hline $3^{\prime}$ & $6.81(\mathrm{~d}, 2.0)$ & 106.8 & $6.84(\mathrm{~d}, 2.0)$ & 106.8 & - & - \\
\hline $1^{\prime \prime}$ & $5.04(\mathrm{~d}, 7.2)$ & 69.8 & $\begin{array}{l}4.56(\mathrm{dd}, 11.2,5.6) \\
4.66(\mathrm{dd}, 11.2,5.6)\end{array}$ & 72.0 & $4.66(\mathrm{~m})$ & 66.0 \\
\hline $2^{\prime \prime}$ & $5.68(\mathrm{dt}, 7.2,1.2)$ & 122.9 & $3.52(\mathrm{t}, 5.6)$ & 59.6 & $5.53(\mathrm{t}, 5.6)$ & 125.1 \\
\hline $3^{\prime \prime}$ & - & 139.6 & - & 73.9 & - & 133.4 \\
\hline $4^{\prime \prime}$ & $2.17(\mathrm{~m})$ & 47.8 & $\begin{array}{l}1.84(\mathrm{dd}, 14.8,5.2) \\
2.11(\mathrm{dd}, 14.8,8.4)\end{array}$ & 41.5 & $\begin{array}{l}2.35(\mathrm{dd}, 17.0,7.2) \\
2.61(\mathrm{dd}, 17.0,5.2)\end{array}$ & 42.6 \\
\hline $5^{\prime \prime}$ & $4.41(\mathrm{~m})$ & 66.2 & $4.92(\mathrm{~m})$ & 72.0 & $5.11(\mathrm{~m})$ & 79.6 \\
\hline $6^{\prime \prime}$ & $5.11(\mathrm{~m})$ & 127.2 & $7.13(\mathrm{t}, 1.6)$ & 148.5 & 7.09 (br t) & 148.9 \\
\hline $7^{\prime \prime}$ & - & 135.1 & - & 130.2 & - & 130.4 \\
\hline $8^{\prime \prime}$ & $1.68(\mathrm{~s})$ & 16.9 & $1.92(\mathrm{t}, 1.6)$ & 10.6 & 1.92 (br s) & 10.6 \\
\hline $9^{\prime \prime}$ & $1.76(\mathrm{~s})$ & 25.7 & $1.39(\mathrm{~s})$ & 18.1 & $1.74(\mathrm{~s})$ & 17.6 \\
\hline $10^{\prime \prime}$ & $1.65(\mathrm{~s})$ & 18.1 & - & 174.2 & - & 177.0 \\
\hline
\end{tabular}




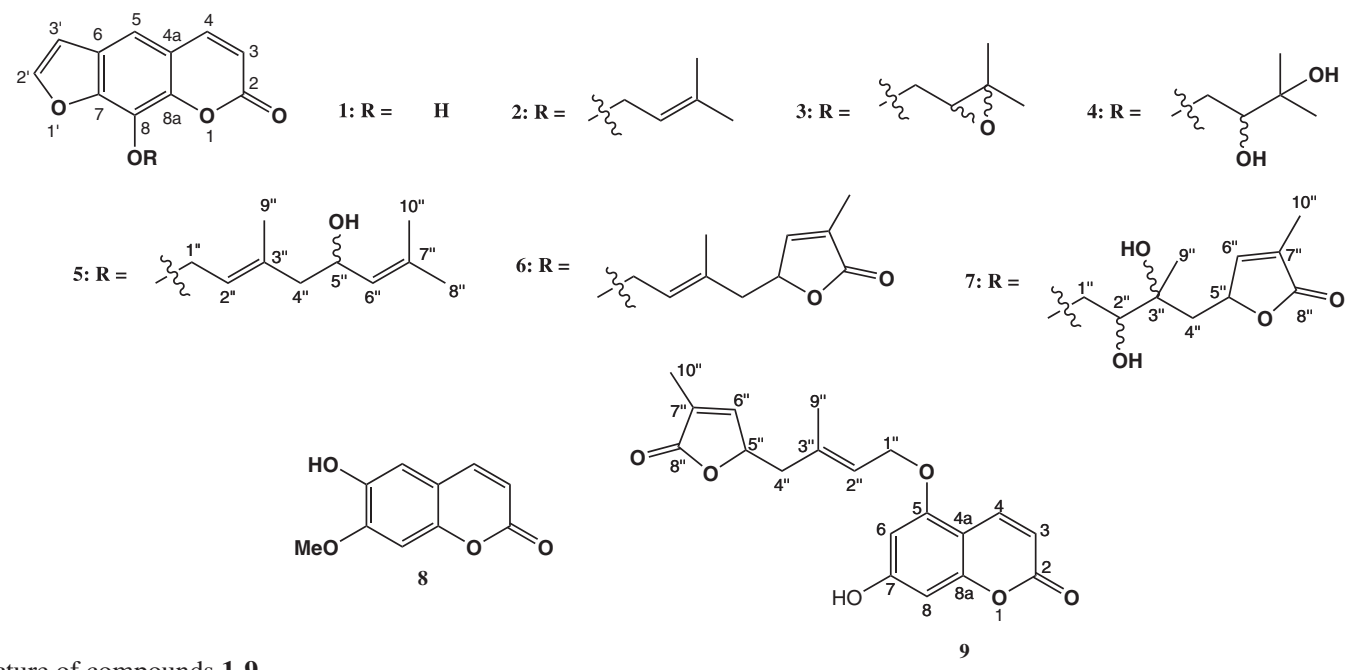

Figure 1. Structure of compounds 1-9.

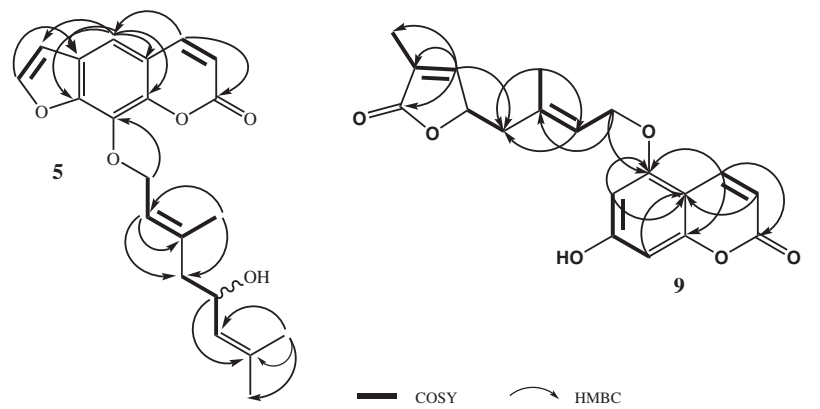

Figure 2. COSY and selective HMBC Correlations of 5 and $\mathbf{9}$.

Table 2. Biological activity of furanocoumarins isolated from the stems of C. lansium

\begin{tabular}{lccc}
\hline Compound & \multicolumn{3}{c}{ Cytotoxicity $\left(\mathrm{IC}_{50}, \mu \mathrm{g} \mathrm{mL}^{-1}\right)$} \\
& $\mathrm{KB}^{\mathrm{a}}$ & $\mathrm{MCF}^{\mathrm{b}}$ & $\mathrm{NCI}-\mathrm{H} 187^{\mathrm{c}}$ \\
\hline $\mathbf{1}$ & 26.97 & 11.92 & 40.41 \\
$\mathbf{2}$ & 9.60 & 37.62 & 28.58 \\
$\mathbf{3}$ & 19.03 & Inactive & 17.40 \\
$\mathbf{5}$ & 22.09 & 31.02 & 28.83 \\
$\mathbf{6}$ & 17.93 & Inactive & 26.23 \\
Doxorubicin & 0.172 & 0.976 & 0.061 \\
\hline
\end{tabular}

${ }^{\mathrm{a}} \mathrm{KB}=$ Oral cavity cancer; ${ }^{\mathrm{b}} \mathrm{MCF} 7=$ Breast cancer; ${ }^{\mathrm{c} N C I}-\mathrm{H} 187=$ Small cell lung cancer

\section{Experimental}

\section{General procedures}

The optical rotation $[\alpha]_{\mathrm{D}}$ values were determined with a Bellingham \& Stanley ADP440 polarimeter. UV-Vis spectra were recorded with a Perkin-Elmer UV-Vis spectrophotometer. The IR spectra were recorded with a Perkin-Elmer FTS FT-IR spectrophotometer. The NMR spectra were recorded using $400 \mathrm{MHz}$ Bruker spectrometer. Chemical shifts were recorded in parts per million $(\delta)$ in $\mathrm{CDCl}_{3}$ with tetramethylsilane (TMS) as an internal reference. The HRMS was obtained from a MicroTOF, Bruker Daltonics or MAT 95 XL mass spectrometers. Quick column chromatography (QCC) and column chromatography (CC) were carried out on silica gel $60 \mathrm{H}$ (Merck, 5-40 $\mu \mathrm{m}$ ) and silica gel 100 (Merck, 63-200 $\mu \mathrm{m})$, respectively. Precoated plates of silica gel $60 \mathrm{~F}_{254}$ were used for analytical purposes.

\section{Plant material}

The twigs of C. lansium were collected in April 2008 from Nan Province, northern part of Thailand. Botanical identification was achieved through comparison with a voucher specimen number QBG 25077 in the herbarium collection of Queen Sirikit Garden, Mae Rim District, Chiang Mai, Thailand.

\section{Extraction and Isolation}

Air-dried twigs of $C$. lansium $(6.73 \mathrm{Kg})$ were successively extracted with $\mathrm{CH}_{2} \mathrm{Cl}_{2}$ and acetone over a period of 3 days each at room temperature. The $\mathrm{CH}_{2} \mathrm{Cl}_{2}$ and acetone extracts were combined (34.02 g) and subjected to QCC over silica gel eluted with a gradient of hexane-acetone (100\% hexane to $100 \%$ acetone) to provide seventeen fractions (A-Q). Fraction $\mathrm{G}(562.9 \mathrm{mg})$ upon standing at room temperature gave compound 2 (247.1 mg). Fraction I (1.83 g) was separated by CC using $20 \%$ EtOAc-hexane to give seven subfractions (I1-I7). Subfraction I7 (546.4 mg) was further purified by $\mathrm{CC}$ with $30 \%$ hexane- $\mathrm{CH}_{2} \mathrm{Cl}_{2}$ yielding compound $\mathbf{3}$ (207.8 $\mathrm{mg})$. Fraction $\mathrm{K}(4.64 \mathrm{~g})$ was separated by $\mathrm{CC}$ eluted with a gradient of $\mathrm{CH}_{2} \mathrm{Cl}_{2}$-EtOAc (5\% EtOAc- $\mathrm{CH}_{2} \mathrm{Cl}_{2}$ to $100 \%$ EtOAc) to give compound $\mathbf{1}(5.6 \mathrm{mg})$, and nine subfractions (K1-K9). Subfraction K6 (124.2 mg) was subjected to 
repeated $\mathrm{CC}$ with $2 \%$ acetone- $\mathrm{CH}_{2} \mathrm{Cl}_{2}$ to afford compound 5 (8.7 mg). Purification of fraction $\mathrm{M}(806.5 \mathrm{mg})$ was performed by sephadex $\mathrm{LH} 20$ with $60 \% \mathrm{CH}_{2} \mathrm{Cl}_{2}-\mathrm{MeOH}$, yielding five subfractions (M1-M5). Subfraction M2 (199.9 mg) was further subjected to repeated $\mathrm{CC}$ with a gradient of $\mathrm{CHCl}_{3}-$ hexane $\left(70 \% \mathrm{CHCl}_{3}\right.$-hexane to $100 \% \mathrm{CHCl}_{3}$ ) to afford eleven subfractions (M2a-M2k). Subfraction M2b (49.9 mg) was further subjected to repeated $\mathrm{CC}$ with $80 \% \mathrm{CHCl}_{3}$-hexane to yield four fractions (M2b1-M2b4). Compound $\mathbf{6}(4.8 \mathrm{mg})$ was derived from fraction M2b2 $(18.5 \mathrm{mg})$ by repeated CC using $60 \% \mathrm{CHCl}_{3}$-hexane whereas compound $7(1.7 \mathrm{mg})$ was obtained from fraction M2b3 $(21.5 \mathrm{mg})$ by repeated $\mathrm{CC}$ with $5 \%$ EtOAc- $\mathrm{CHCl}_{3}$. Subfraction M2d $(8.6 \mathrm{mg}$ ) was further purified by prep.TLC with $5 \%$ EtOAc- $\mathrm{CHCl}_{3}$ to afford $6(7.3 \mathrm{mg})$. Fraction N (621.1 mg) was performed by $\mathrm{CC}$ with $40 \%$ EtOAc-hexane, yielding eleven subfractions (N1-N11). Subfraction N8 (65.5 mg) was separated by CC using a gradient of EtOAc- $\mathrm{CH}_{2} \mathrm{Cl}_{2}\left(10 \%\right.$ EtOAc- $\mathrm{CH}_{2} \mathrm{Cl}_{2}$ to $25 \%$ EtOAc- $\left.\mathrm{CH}_{2} \mathrm{Cl}_{2}\right)$ to yield compound $9(4.0 \mathrm{mg})$ while subfraction N10 (39.4 mg) was purified by prep.TLC with $7 \%$ EtOAc- $\mathrm{CH}_{2} \mathrm{Cl}_{2}$ to give compound 4 (9.8 mg).

\section{Clausenalansimin A (5)}

Yellow viscous oil; $[\alpha]_{\mathrm{D}}^{27}+48.1^{\circ}\left(c 0.02, \mathrm{CHCl}_{3}\right) ; \mathrm{UV} \lambda_{\max } / \mathrm{nm}$ (MeOH): 204, 216, 247, 299; IR (neat) $v_{\max } / \mathrm{cm}^{-1}: 3442,2924$, 2855, 1722, 1626, 1587, 1443, 1401, 1327, 1261, 1150, 1092, 1026, 870, 801, 754; ${ }^{1} \mathrm{H} \mathrm{NMR}\left(\mathrm{CDCl}_{3}, 400 \mathrm{MHz}\right)$ and ${ }^{13} \mathrm{C}$ NMR $\left(\mathrm{CDCl}_{3}, 100 \mathrm{MHz}\right)$, see Table 1; HRMS (APCI, -ve) $\mathrm{m} / z: 389.1161$ ([M+Cl], calc. $\left.\mathrm{C}_{21} \mathrm{H}_{22} \mathrm{O}_{5} \mathrm{Cl}, 389.1156\right)$.

\section{Clausenalansimin B (9)}

Yellow viscous oil; $[\alpha]_{\mathrm{D}}^{27}+17.24^{\circ}\left(c 0.02, \mathrm{CHCl}_{3}\right)$; UV $\lambda_{\max } / \mathrm{nm}(\mathrm{MeOH}): 207$ and 329; IR (neat) $v_{\max } / \mathrm{cm}^{-1}: 2921$, 1726, 1609, 1454, 1364, 1237, 1154, 1115, 823, 612; ${ }^{1} \mathrm{H}$ NMR $\left(\mathrm{CDCl}_{3}, 400 \mathrm{MHz}\right)$ and ${ }^{13} \mathrm{CNMR}\left(\mathrm{CDCl}_{3}, 100 \mathrm{MHz}\right)$ : see Table 1; HREIMS $m / z: 342.1158$ ([M] ${ }^{+}$, calc. $\mathrm{C}_{19} \mathrm{H}_{18} \mathrm{O}_{6}, 342.1103$ ).

\section{Cytotoxicity assay}

The procedures for cytotoxic assay were performed by sulphorhodamine B (SRB) assay (anti-KB and MCF7) and colorimetric method (anti-NCI-H187) as described by Skehan et al. ${ }^{6}$ In this study, three cancer cell lines, MCF7 (breast cancer), NCI-H187 (human, small cell lung cancer) and KB (oral human epidermal carcinoma) were used. Doxorubicin was the reference substance in this study.

\section{Supplementary Information}

Supplementary data are available free of charge at http://jbcs.sbq.org.br, as PDF file.

\section{Acknowledgments}

We would like to thank the Thailand Research Fund (Grant no. RSA5280011) and Mae Fah Luang University for financial support and to the Bioassay Research Facility of BIOTEC (Thailand) for cytotoxicity tests. WM thanks Mae Fah Luang University for a Ph.D graduate student research grant. We are indebted to Mr. Nitirat Chimnoi, Chulabhorn Research Institute, Bangkok, for recording mass spectrum and Ms. Nareerat Tongtip, Department of Chemistry, Faculty of Science, Phuket Rajabhat University for recording NMR spectral data. We also thank Assoc. Prof. Dr Chatchanok Karalai and Assoc. Prof. Chanita Ponglimanont, Department of Chemistry, Faculty of Science, Prince of Songkla University for supporting some scientific material and giving valuable suggestions.

\section{References}

1. Adebajo, A. C.; Iwalewa, E. O.; Obuotor, E. M.; Ibikunle, G. F.; Omisore, N. O.; Adewunmi, C. O.; Obaparusi, O. O.; Klaes, M.; Adetogun, G. E.; Schmidt, T. J.; Verspohl, E. J.; J. Ethnopharmacol. 2009, 10, 122.

2. Lin, J. H.; Phytochemistry 1989, 28, 621.

3. Ng, T. B.; Lam, S. K.; Fong, W. P.; Biol. Chem. 2003, 384, 289.

4. Yang, M. H.; Chen, Y. Y.; Huang, L.; Phytochemistry 1988, $27,445$.

5. Kumar, V.; Vallipuram, K.; Adebajo, A. C.; Reisch, J.; Phytochemistry 1995, 40, 1563.

6. Skehan, P.; Storeng, R.; Scudiero, D.; Monks, A.; McMahon, J.; Vistica, D.; Warren, J. T.; Bokesch, H.; Kenney, S.; Boyd, R. M.; J. Natl. Cancer Inst. 1990, 82, 1107.

7. Ito, C.; Katsuno, S.; Furukawa, H.; Chem. Pharm. Bull. 1998, 46, 341.

8. Gellért, M.; Reisch, J.; Szendrei, K.; Novák, I.; Csedö, K.; Phytochemistry 1972, 11, 2894.

9. Ngadjui, B. T.; Ayafor, J. F.; Sondengam B. L.; Connolly, J. D.; Phytochemistry 1989, 28, 585.

10. Nakamura, K.; Takemura, Y.; Ju-ichi, M.; Ito, C.; Furukawa, H.; Heterocycles 1998, 43, 549.

11. Masuda, T.; Takasugi, M.; Anetai, M.; Phytochemistry 1998, 47, 13.

12. Adityachaudhury, N.; Ghosh, D.; Choudhuri, A.; Phytochemistry 1974, 13, 235.

13. Razdan, T. K.; Kachroo, V.; Harkar, S.; Koul, S.; Phytochemistry 1982, 21, 923.

14. Khan, N. U.; Navqi, S. W. I.; Ishratullah, K.; Phytochemistry 1983, 22, 2624.

15. Rakash, D.; Raj, K.; Kapil, R. S.; Popli, S. P.; Phytochemistry 1978, 17, 1194.

16. Al-Barwani, F. M.; Eltayeb, E. A.; Biochem. Syst. Ecol. 2004, 32, 1097.

Received: July 18, 2009

Web Release Date: December 16, 2009 


\section{New Coumarins from Clausena lansium Twigs}

\section{Wisanu Maneerat, ${ }^{a}$ Uma Prawat, $^{b}$ Nisakorn Saewan ${ }^{c}$ and Surat Laphookhieo ${ }^{*, a}$}

${ }^{a}$ Natural Products Research Laboratory, School of Science, Mae Fah Luang University, Tasud, Muang, 57100 Chiang Rai, Thailand

${ }^{b}$ Faculty of Science and Technology, Phuket Rajabhat University, Muang, 83000 Phuket, Thailand

${ }^{c}$ School of Cosmetic Science, Mae Fah Luang University, Tasud, Muang,

57100 Chiang Rai, Thailand

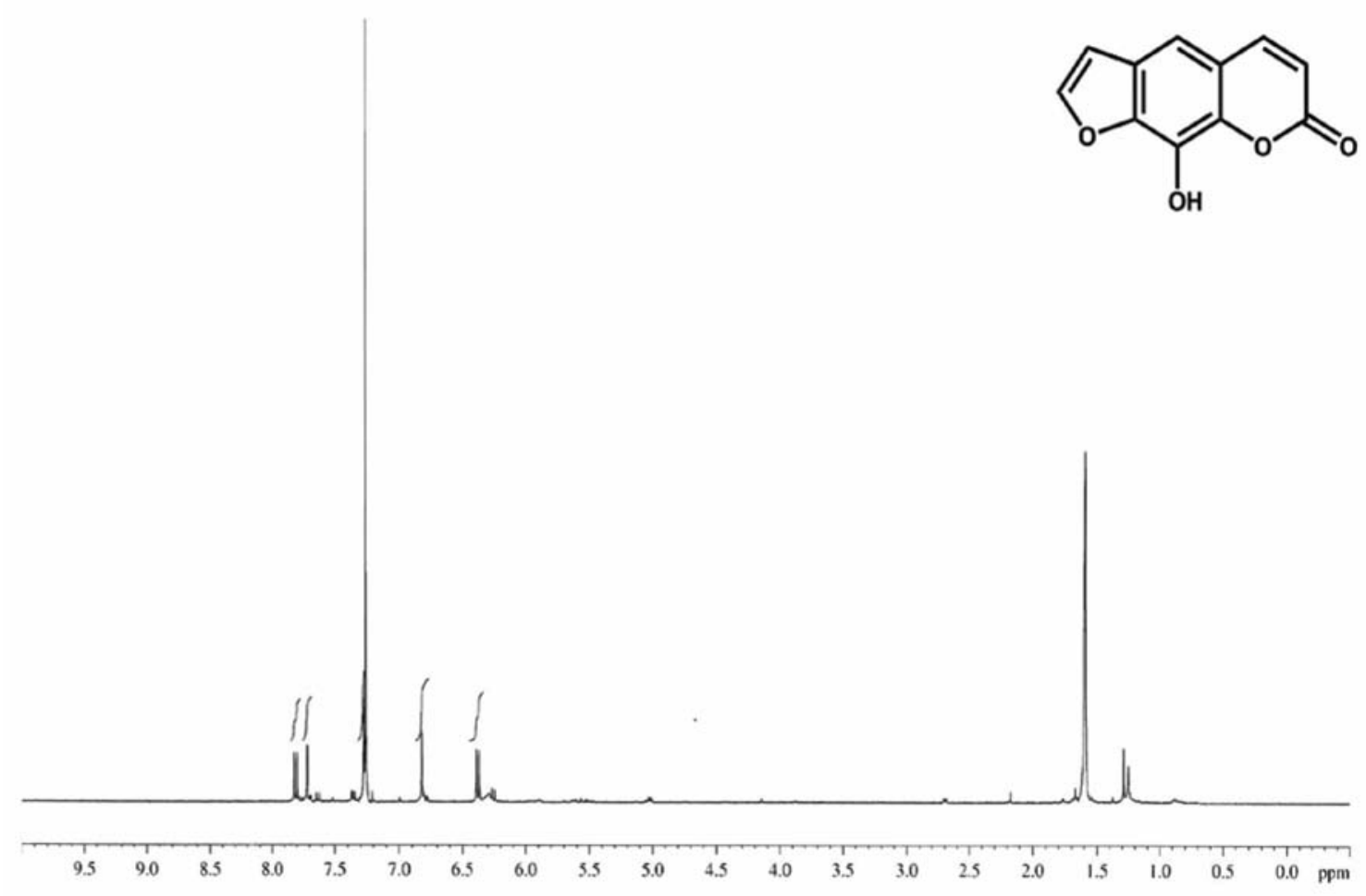

Figure S1. ${ }^{1} \mathrm{H}$ NMR spectrum of xanthotoxol (1) $\left(400 \mathrm{MHz}, \mathrm{CDCl}_{3}\right)$. 

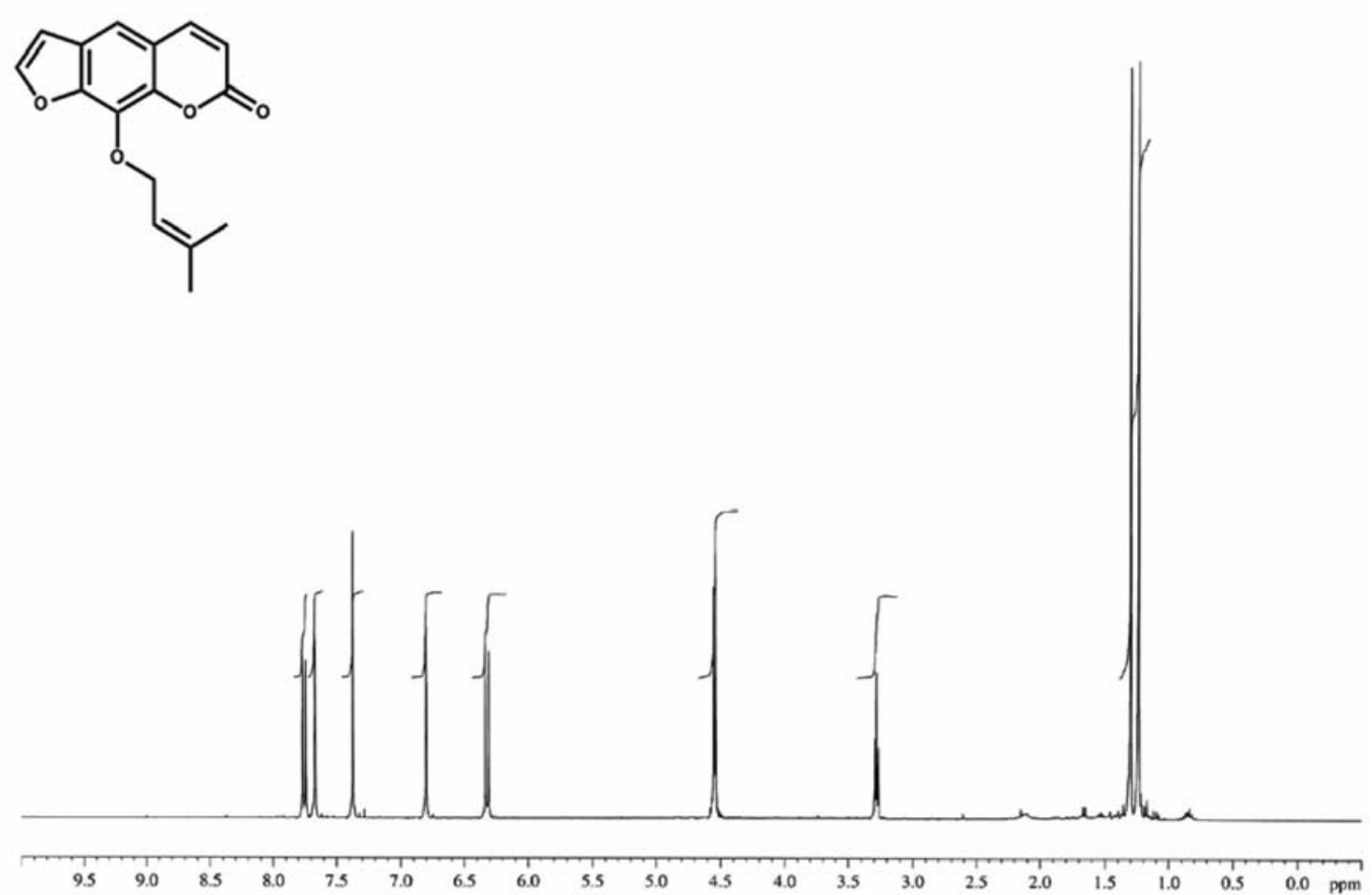

Figure S2. ${ }^{1} \mathrm{H}$ NMR spectrum of imperatorin (2) (400 MHz, $\left.\mathrm{CDCl}_{3}\right)$.
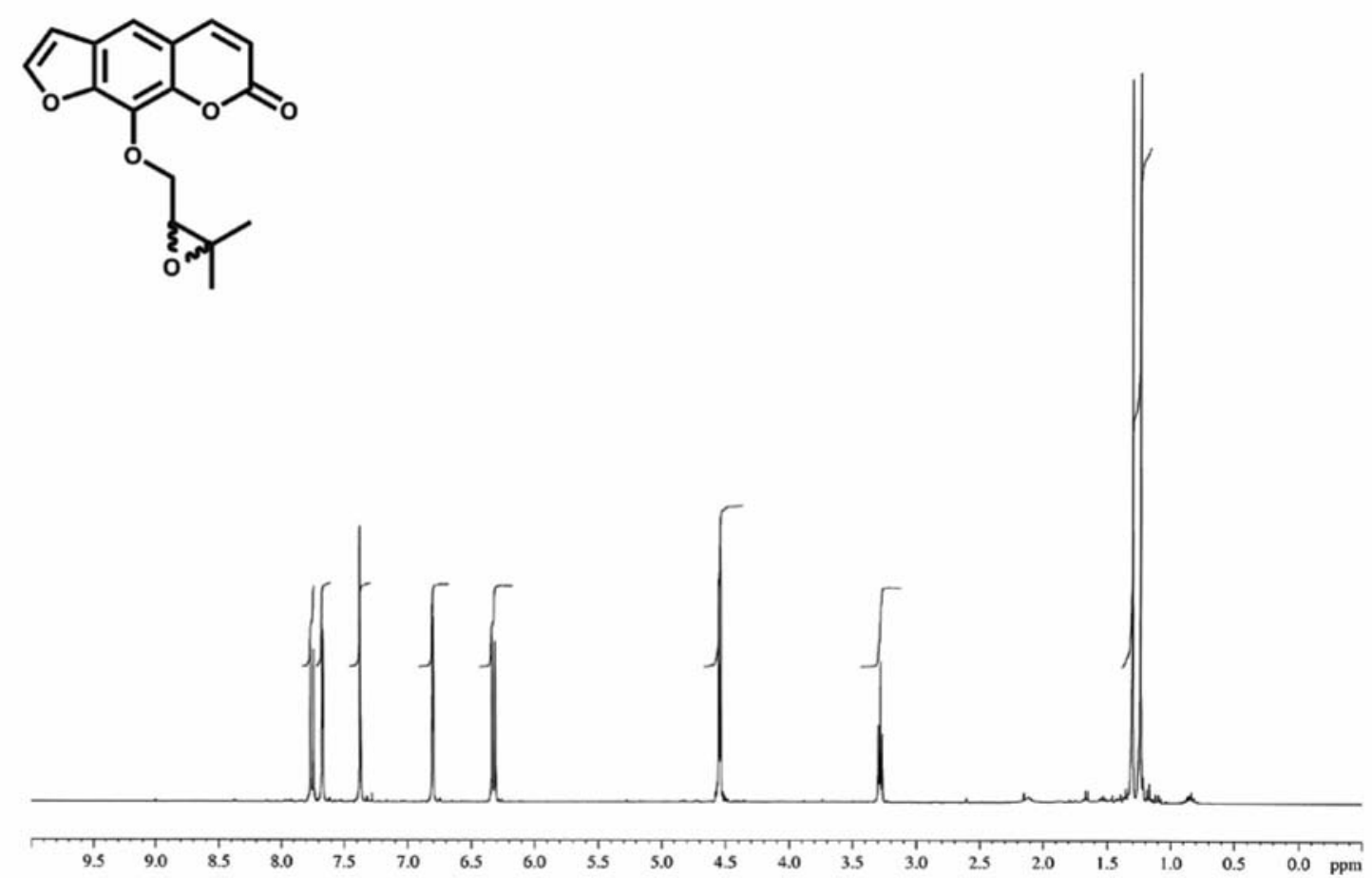

Figure S3. ${ }^{1} \mathrm{H}$ NMR spectrum of heraclenin (3) $\left(400 \mathrm{MHz}, \mathrm{CDCl}_{3}\right)$. 

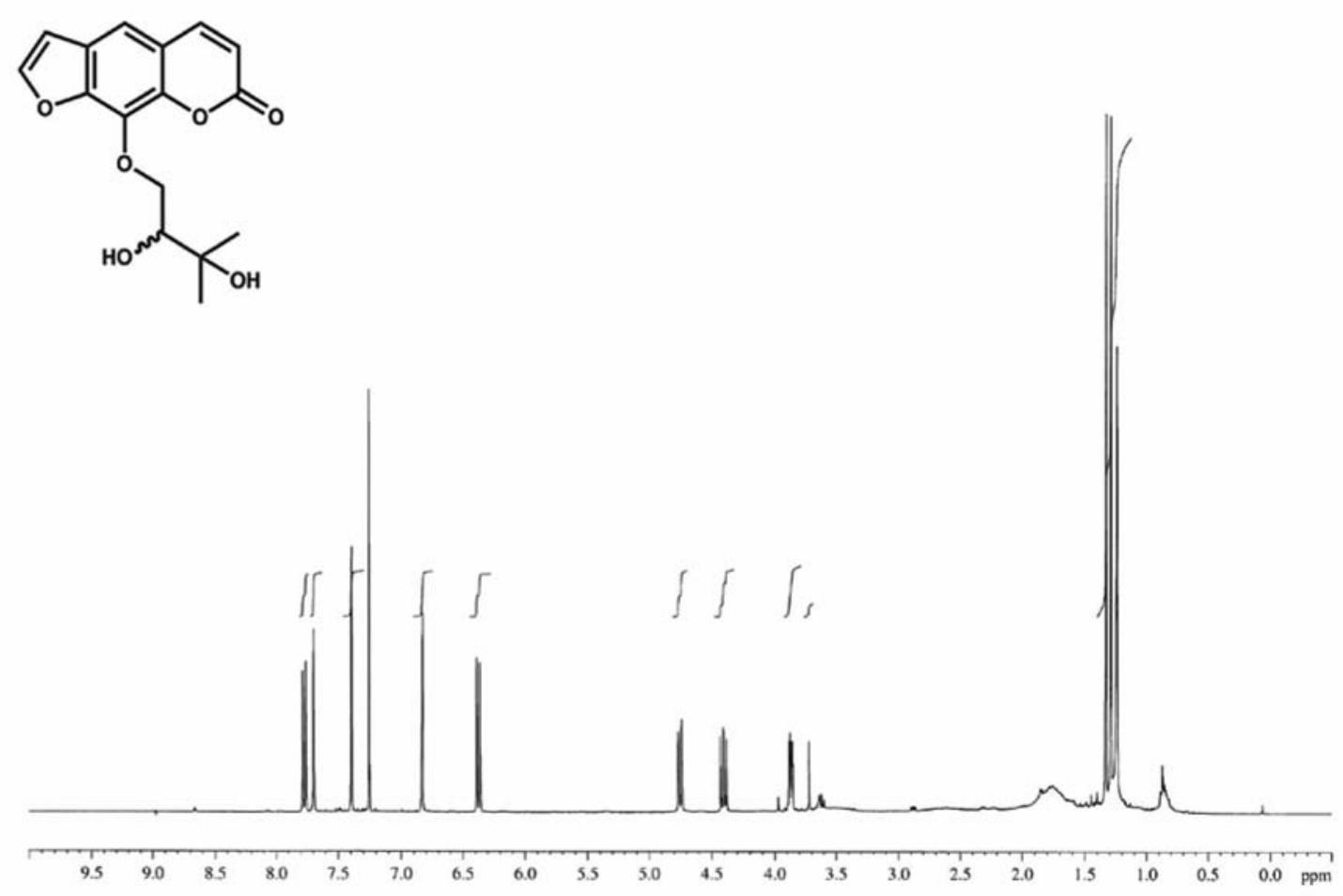

Figure S4. ${ }^{1} \mathrm{H}$ NMR spectrum of heraclenol (4) $\left(400 \mathrm{MHz}, \mathrm{CDCl}_{3}\right)$.
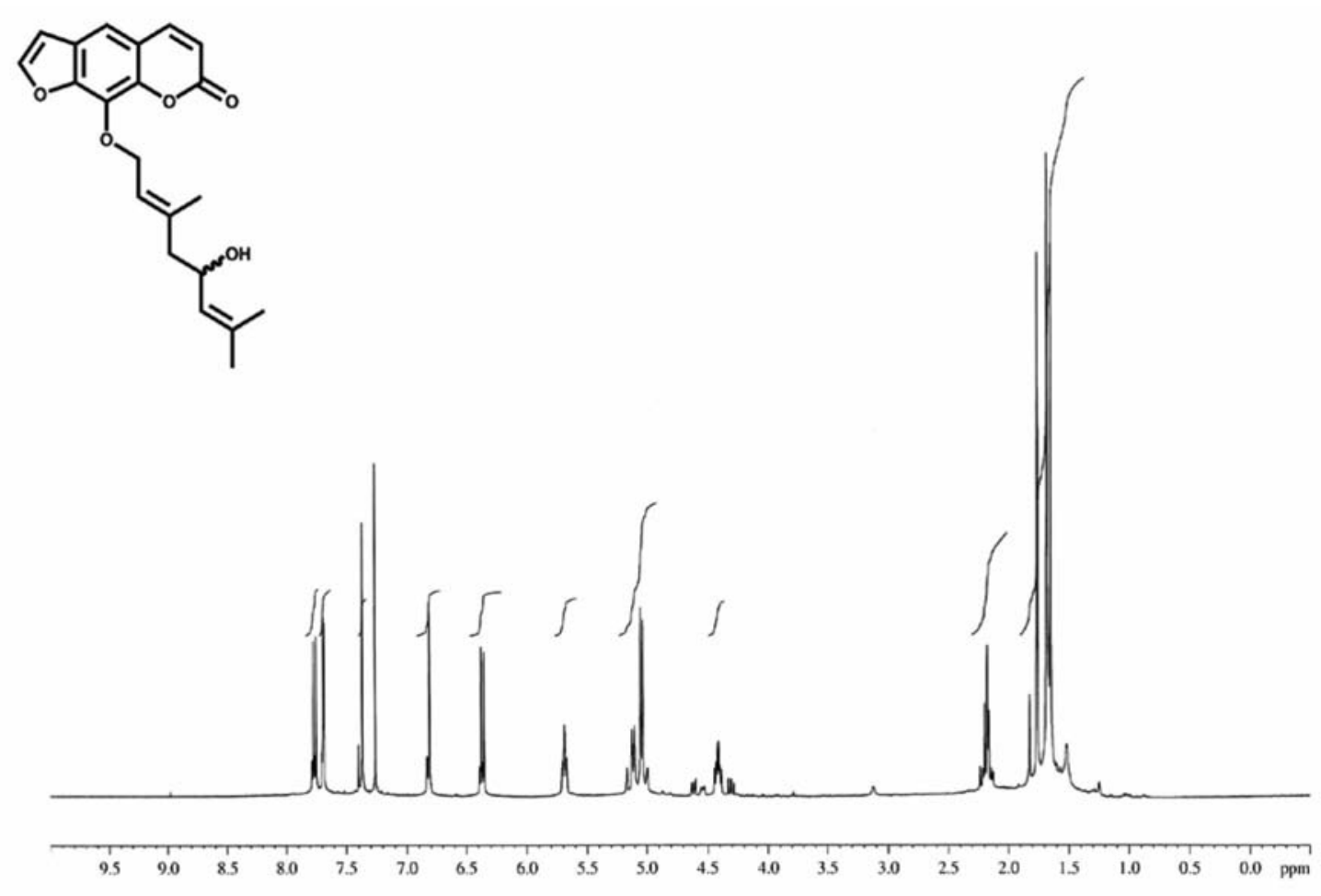

Figure S5. ' $\mathrm{H}$ NMR spectrum of clausenalansimin A (5) $\left(400 \mathrm{MHz}, \mathrm{CDCl}_{3}\right)$. 


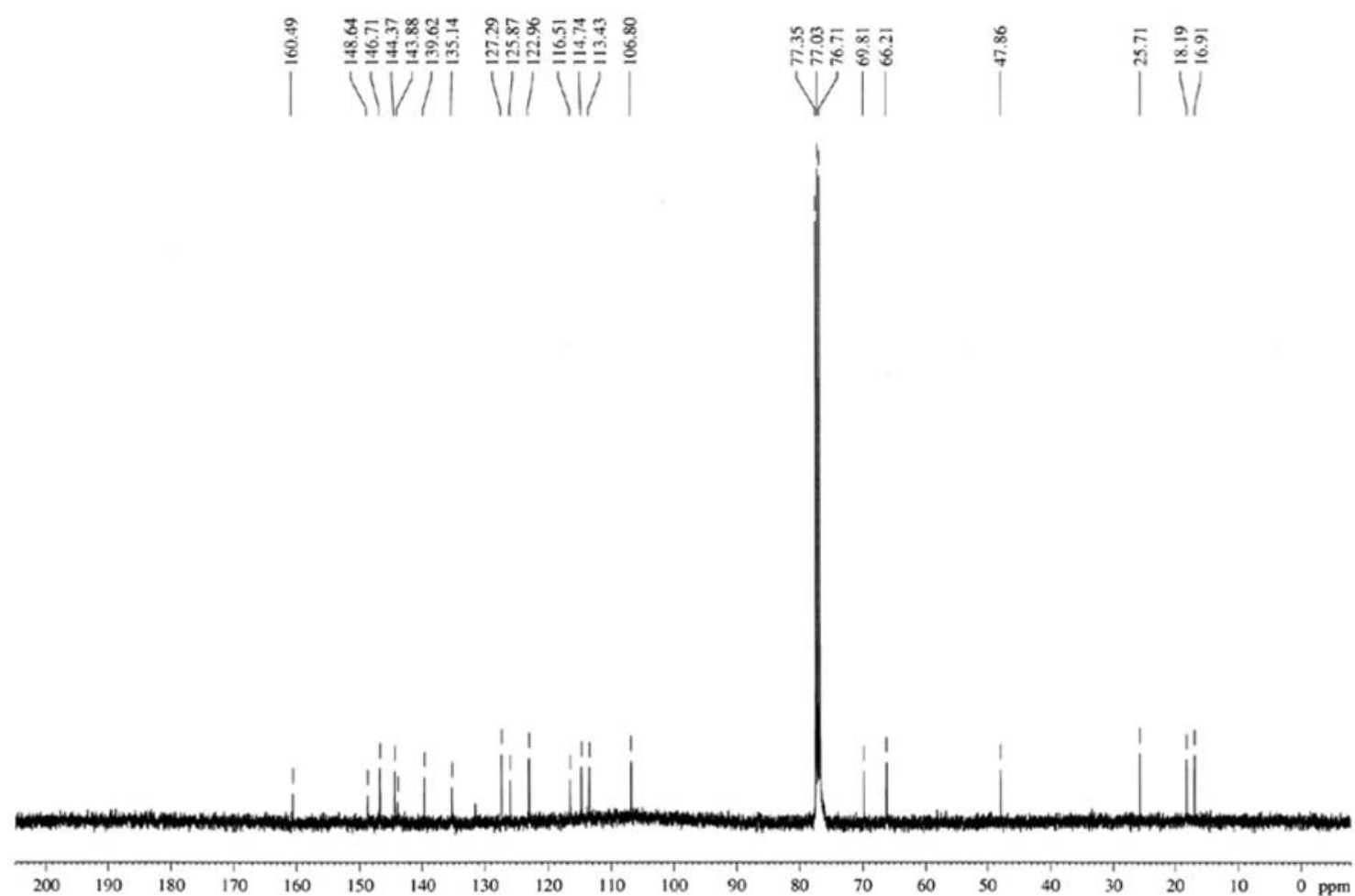

Figure S6. ${ }^{13} \mathrm{C}$ NMR spectrum of clausenalansimin A (5) (100 MHz, $\left.\mathrm{CDCl}_{3}\right)$.
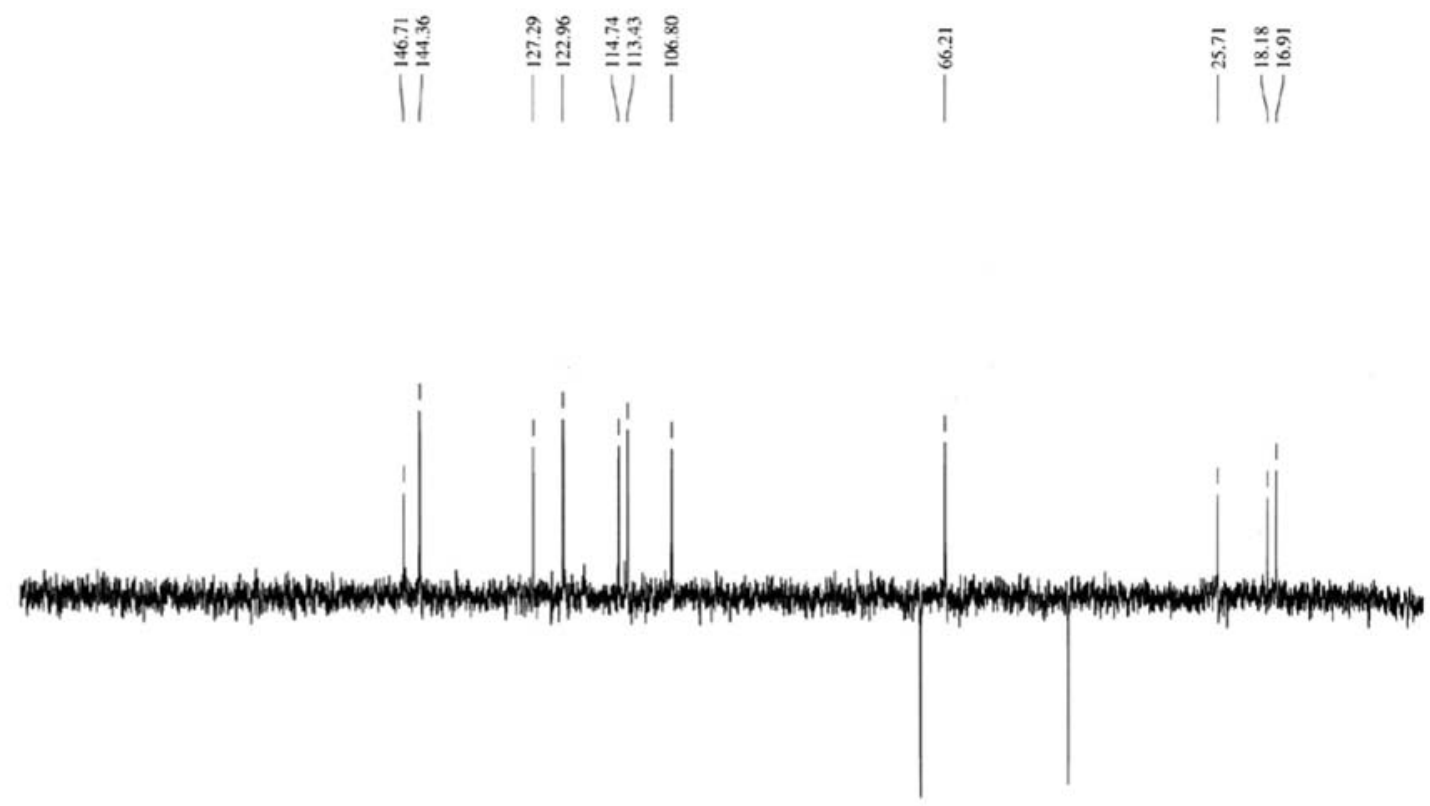

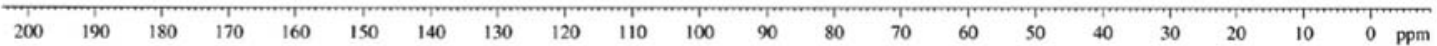

Figure S7. DEPT $135^{\circ}$ spectrum of clausenalansimin A (5) $\left(100 \mathrm{MHz}, \mathrm{CDCl}_{3}\right)$. 
Vol. 21, No. 4, 2010

Maneerat et al.

S5

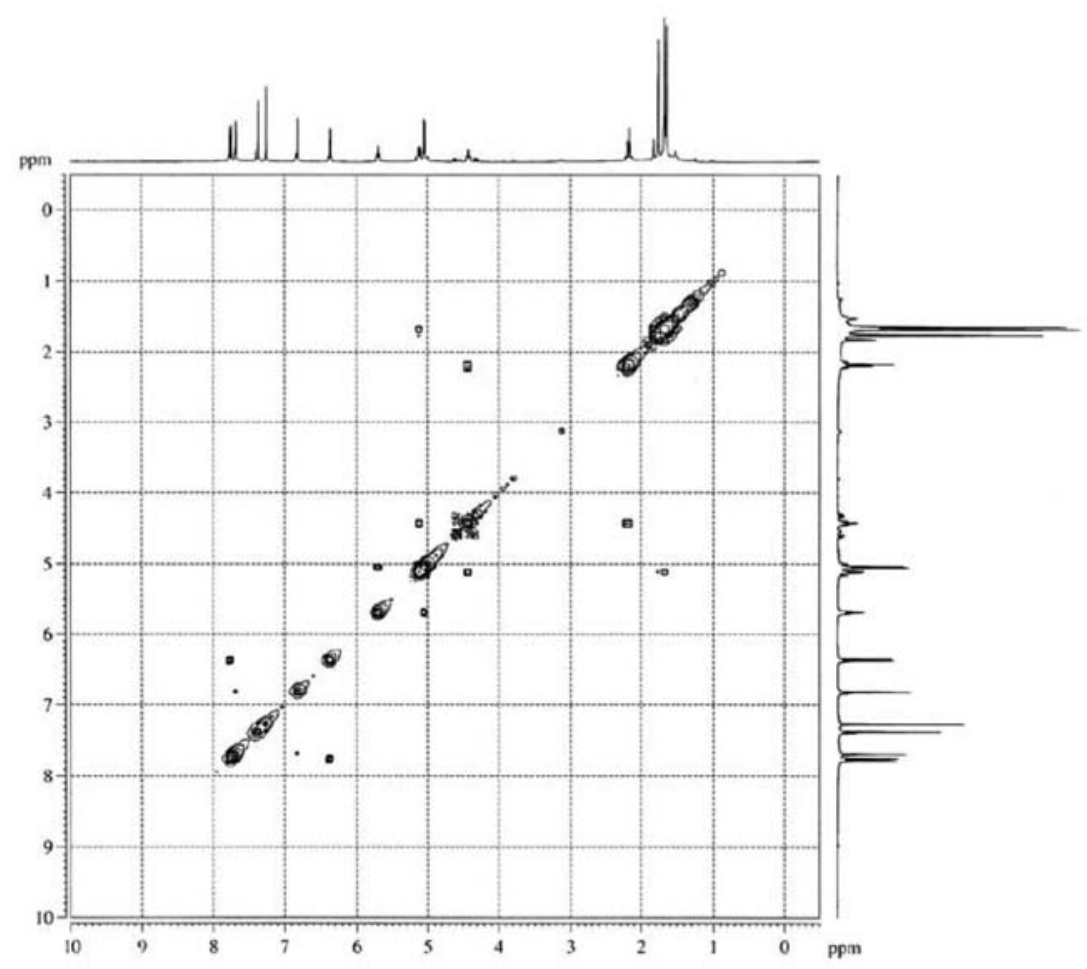

Figure S8. COSY spectrum of clausenalansimin A (5) $\left(400 \mathrm{MHz}, \mathrm{CDCl}_{3}\right)$.

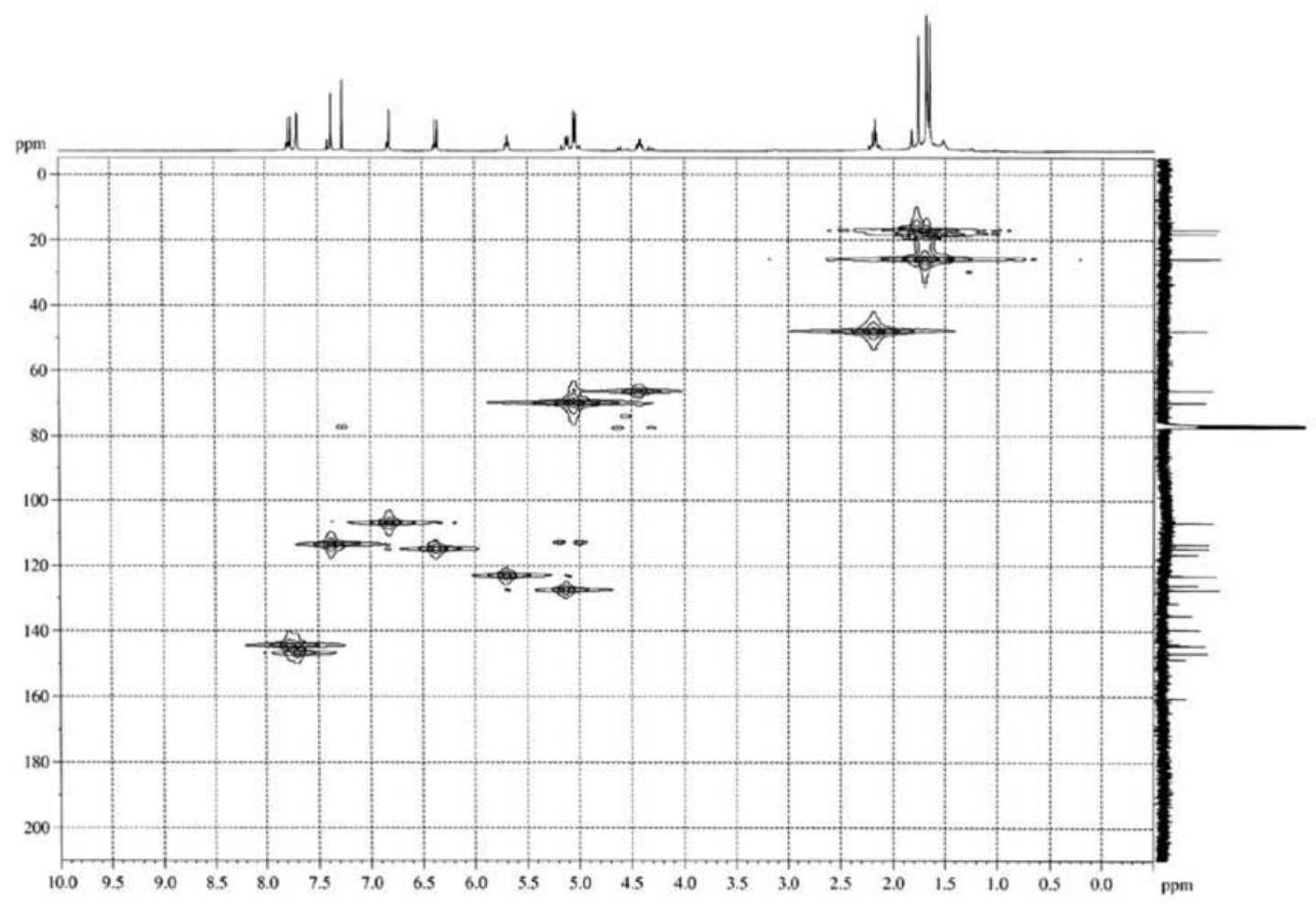

Figure S9. HMQC spectrum of clausenalansimin A (5) $\left(400 \mathrm{MHz}, \mathrm{CDCl}_{3}\right)$. 


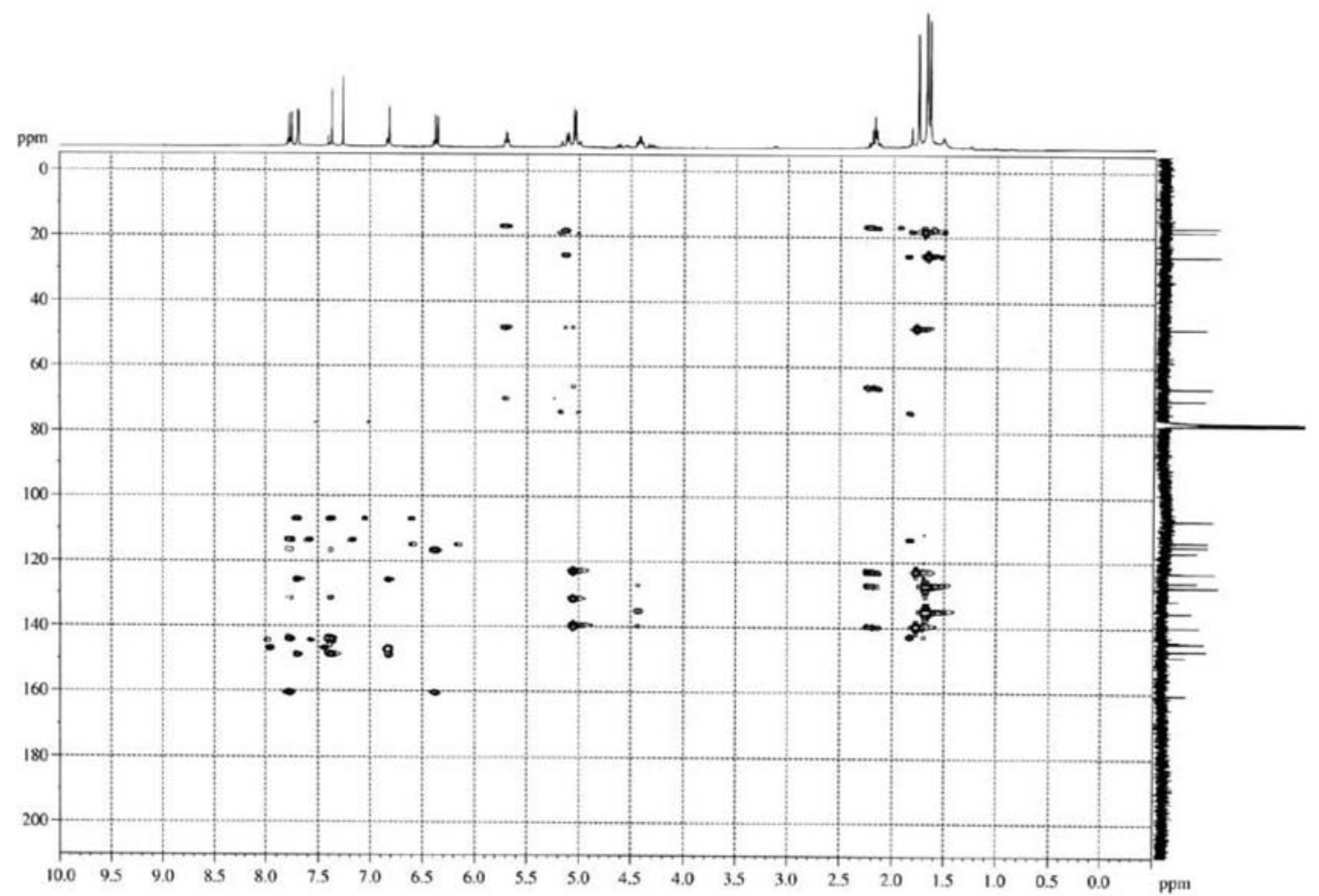

Figure S10. HMBC spectrum of clausenalansimin A (5) (400 MHz, $\left.\mathrm{CDCl}_{3}\right)$.

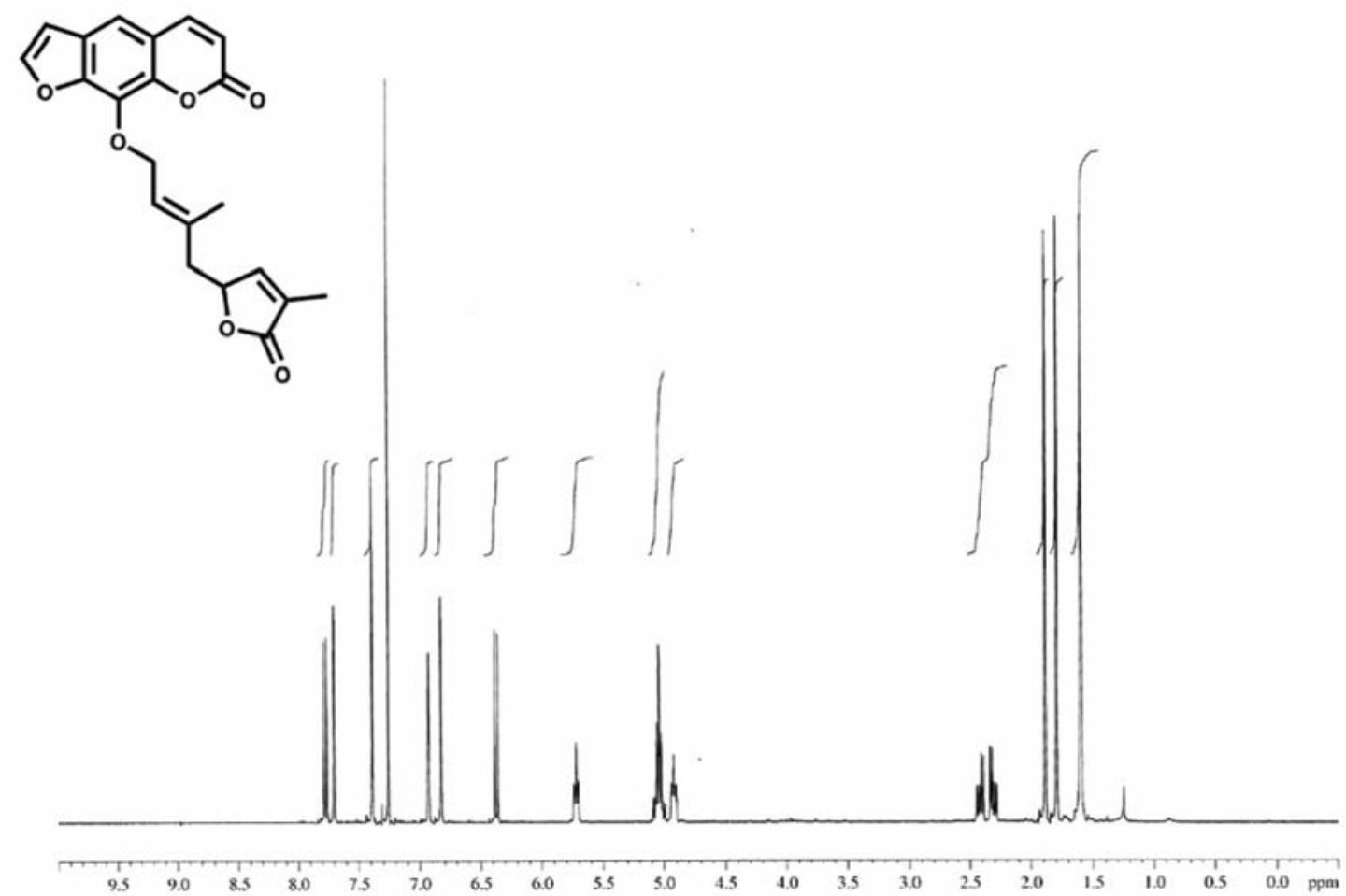

Figure S11. ${ }^{1} \mathrm{H}$ NMR spectrum of wampetin (6) $\left(400 \mathrm{MHz}, \mathrm{CDCl}_{3}\right)$. 

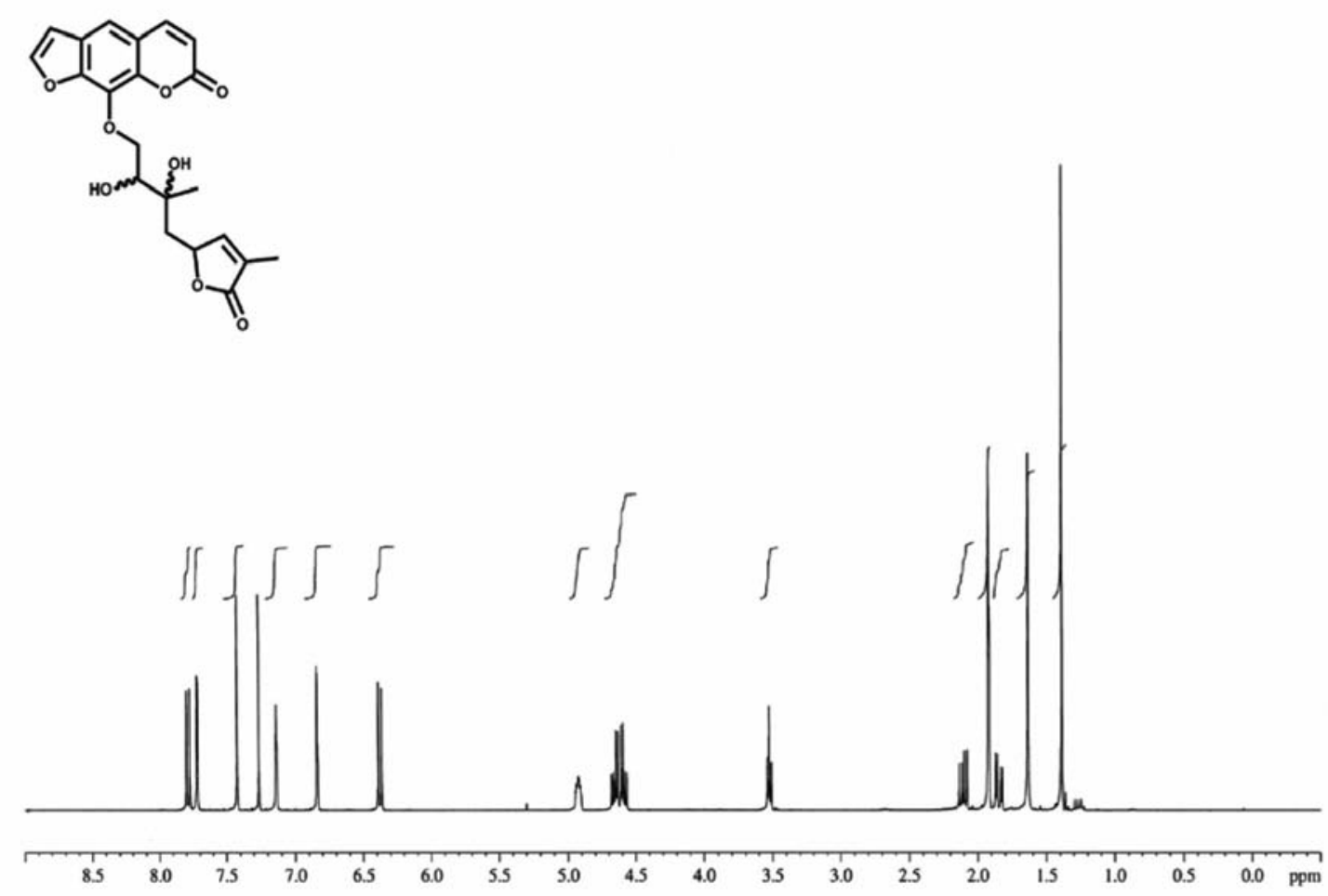

Figure S12. ${ }^{1} \mathrm{H}$ NMR spectrum of indicolactonediol (7) (400 $\mathrm{MHz}, \mathrm{CDCl}_{3}$ ).

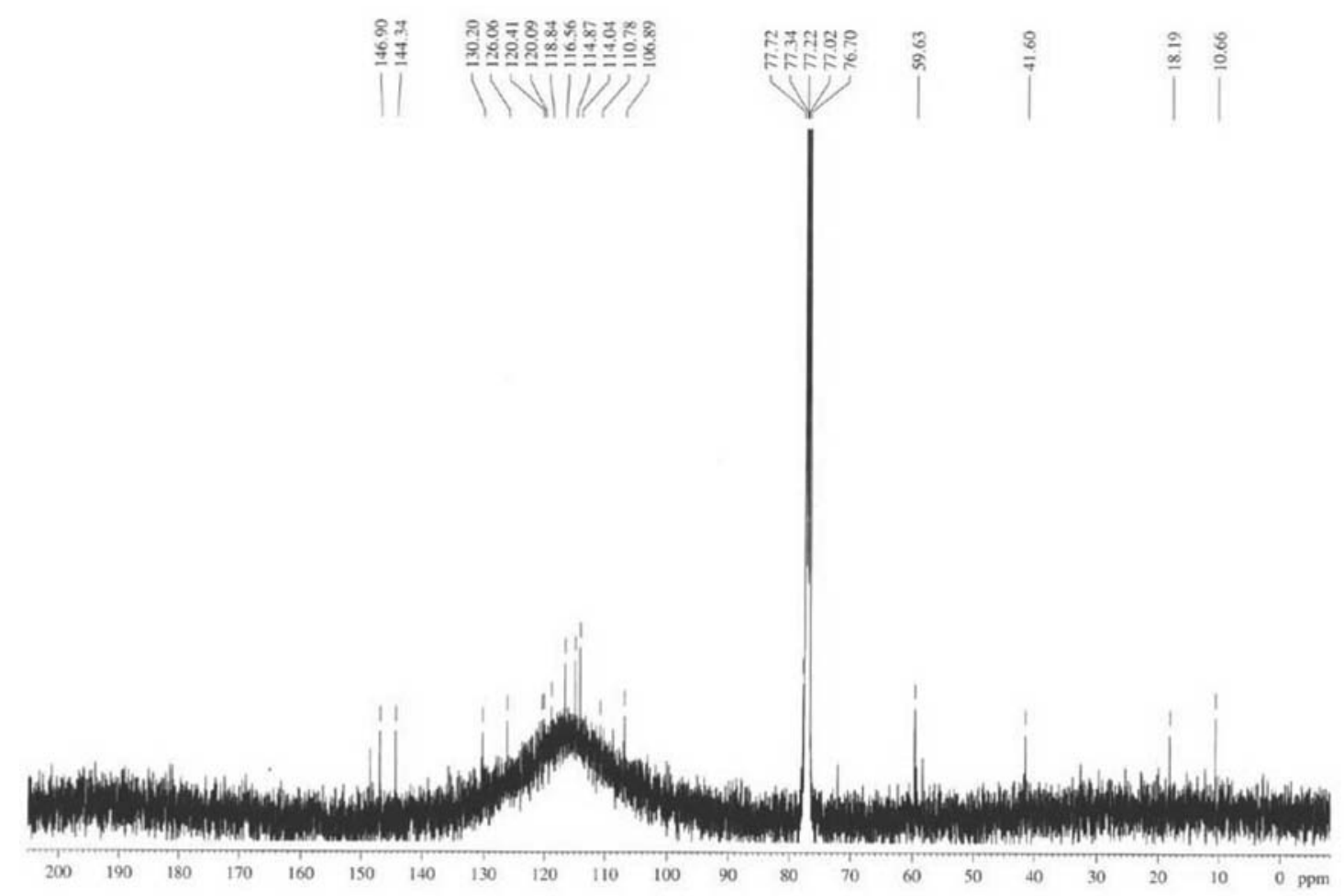

Figure S13. ${ }^{13} \mathrm{C}$ NMR spectrum of indicolactonediol (7) (100 $\mathrm{MHz}, \mathrm{CDCl}_{3}$ ). 


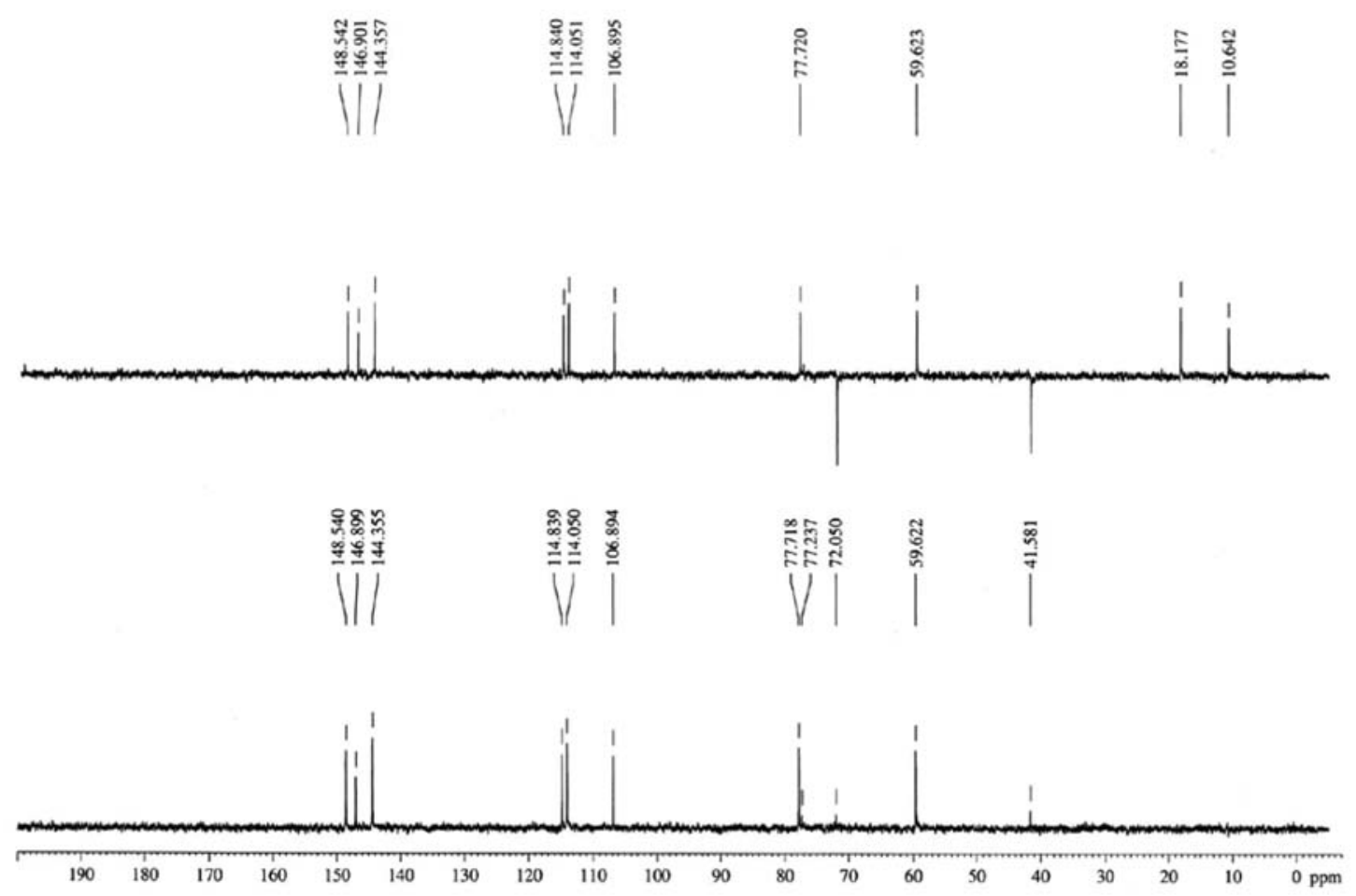

Figure S14. DEPT $135^{\circ}$ and $90^{\circ}$ spectrum of indicolactonediol (7) $\left(100 \mathrm{MHz}, \mathrm{CDCl}_{3}\right)$.

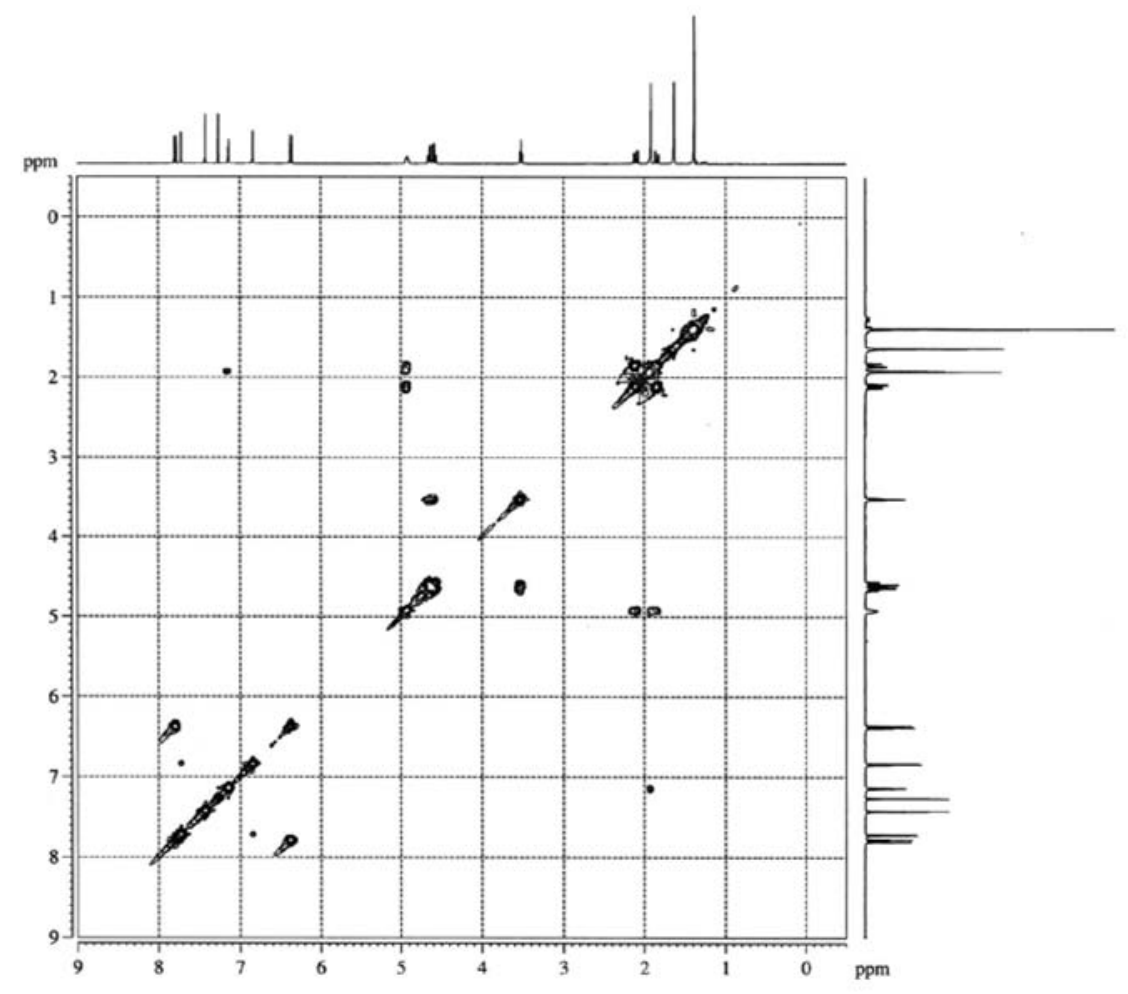

Figure S15. COSY spectrum of indicolactonediol (7) $\left(400 \mathrm{MHz}, \mathrm{CDCl}_{3}\right)$. 


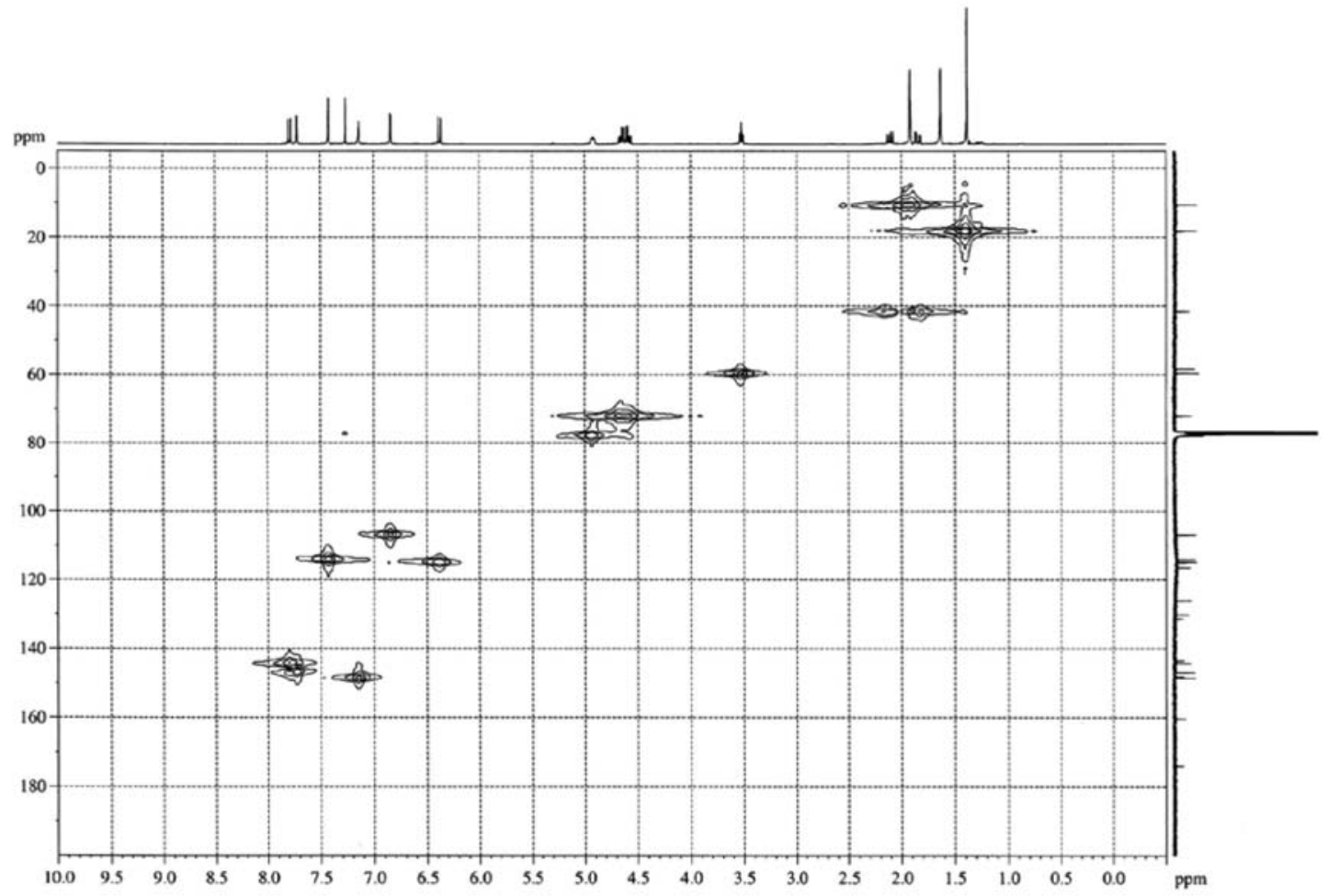

Figure S16. HMQC spectrum of indicolactonediol (7) (400 MHz, $\left.\mathrm{CDCl}_{3}\right)$.

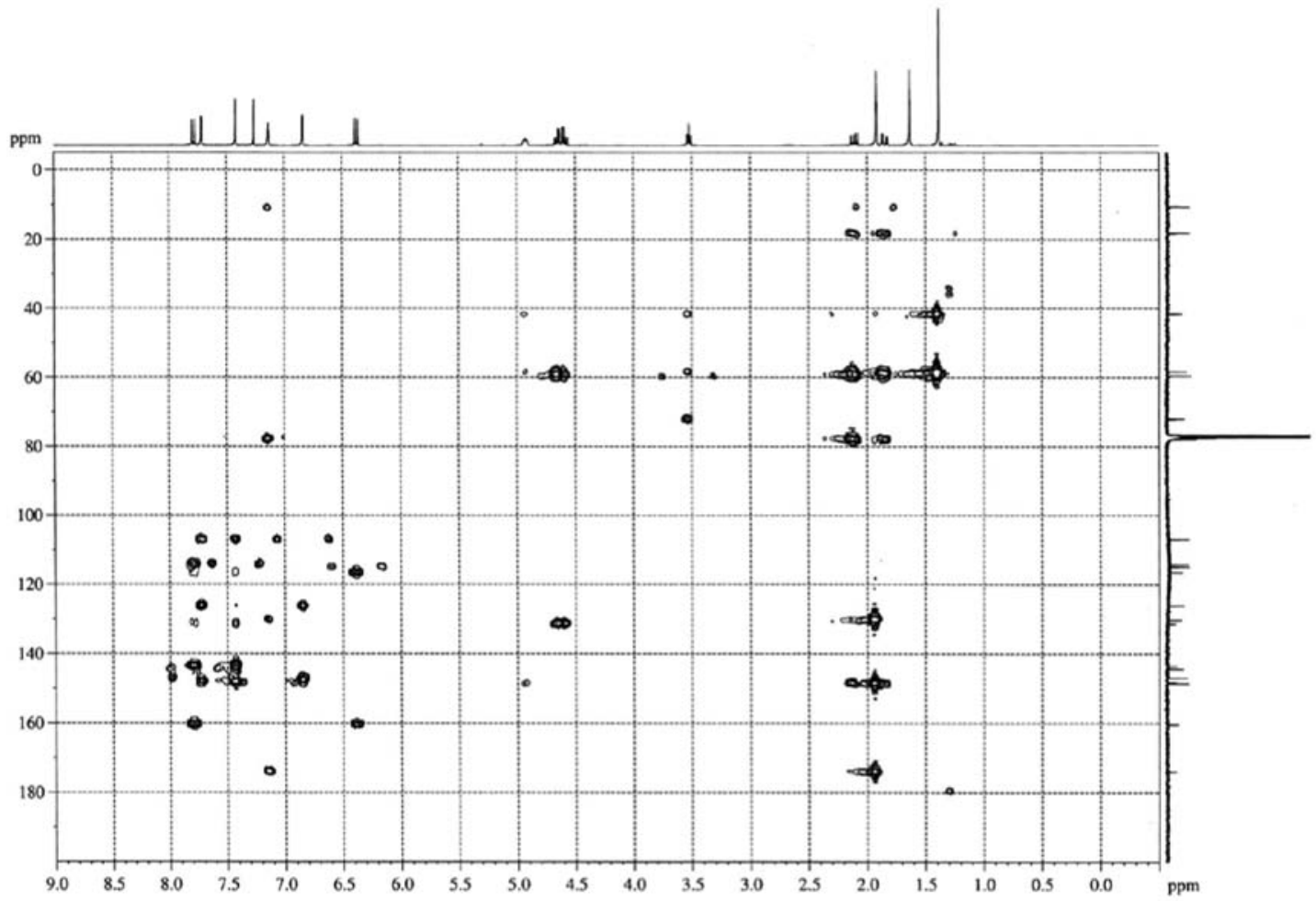

Figure S17. HMBC spectrum of indicolactonediol (7) (400 MHz, $\mathrm{CDCl}_{3}$ ). 

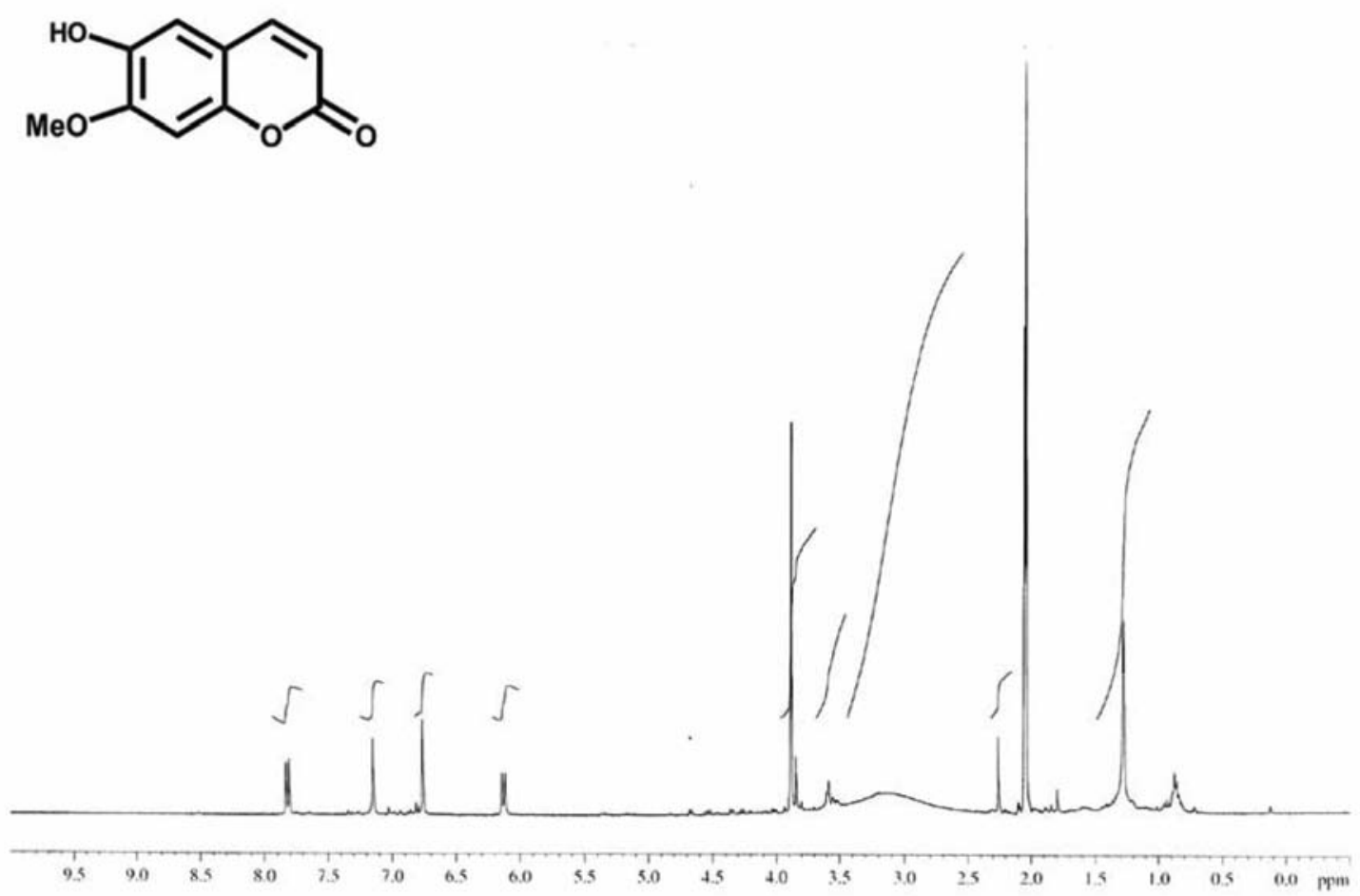

Figure S18. ${ }^{1} \mathrm{H}$ NMR spectrum of indicolactonediol (8) (400 MHz, Acetone-d 6 ).
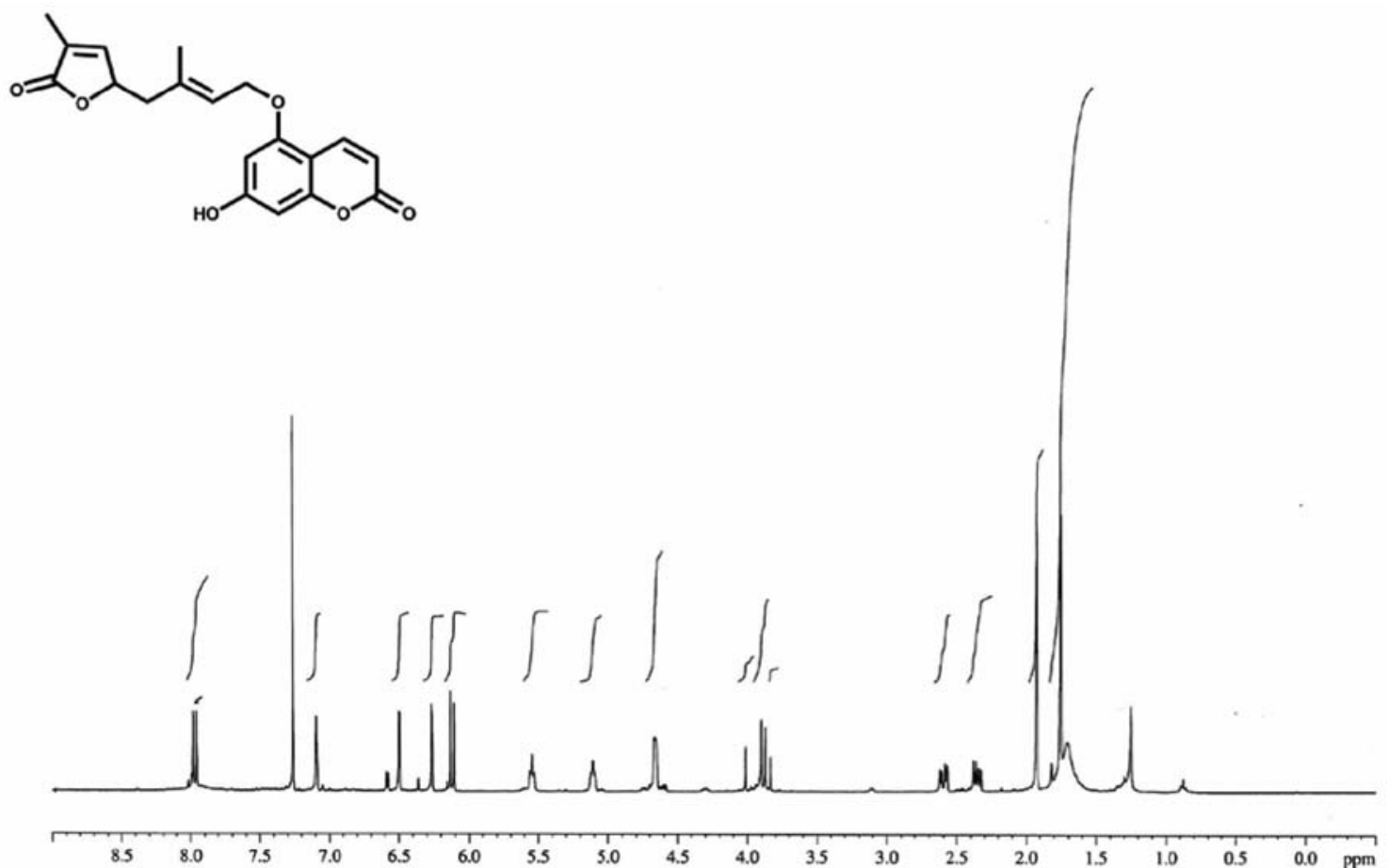

Figure S19. ${ }^{1} \mathrm{H}$ NMR spectrum of clausenalansimin B (9) (400 $\left.\mathrm{MHz}, \mathrm{CDCl}_{3}\right)$. 


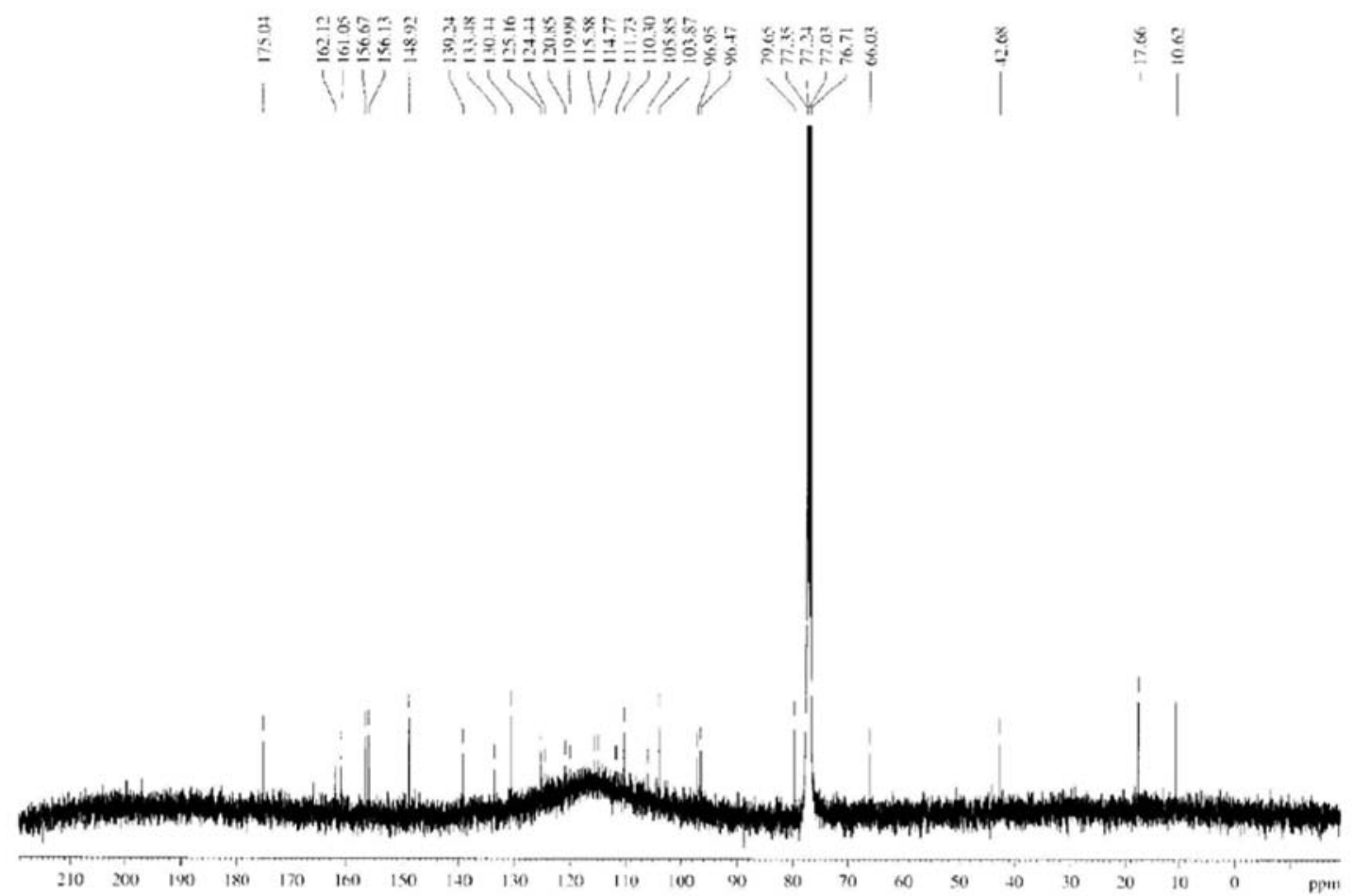

Figure S20. ${ }^{13} \mathrm{C}$ NMR spectrum of clausenalansimin B (9) $\left(100 \mathrm{MHz}, \mathrm{CDCl}_{3}\right)$.
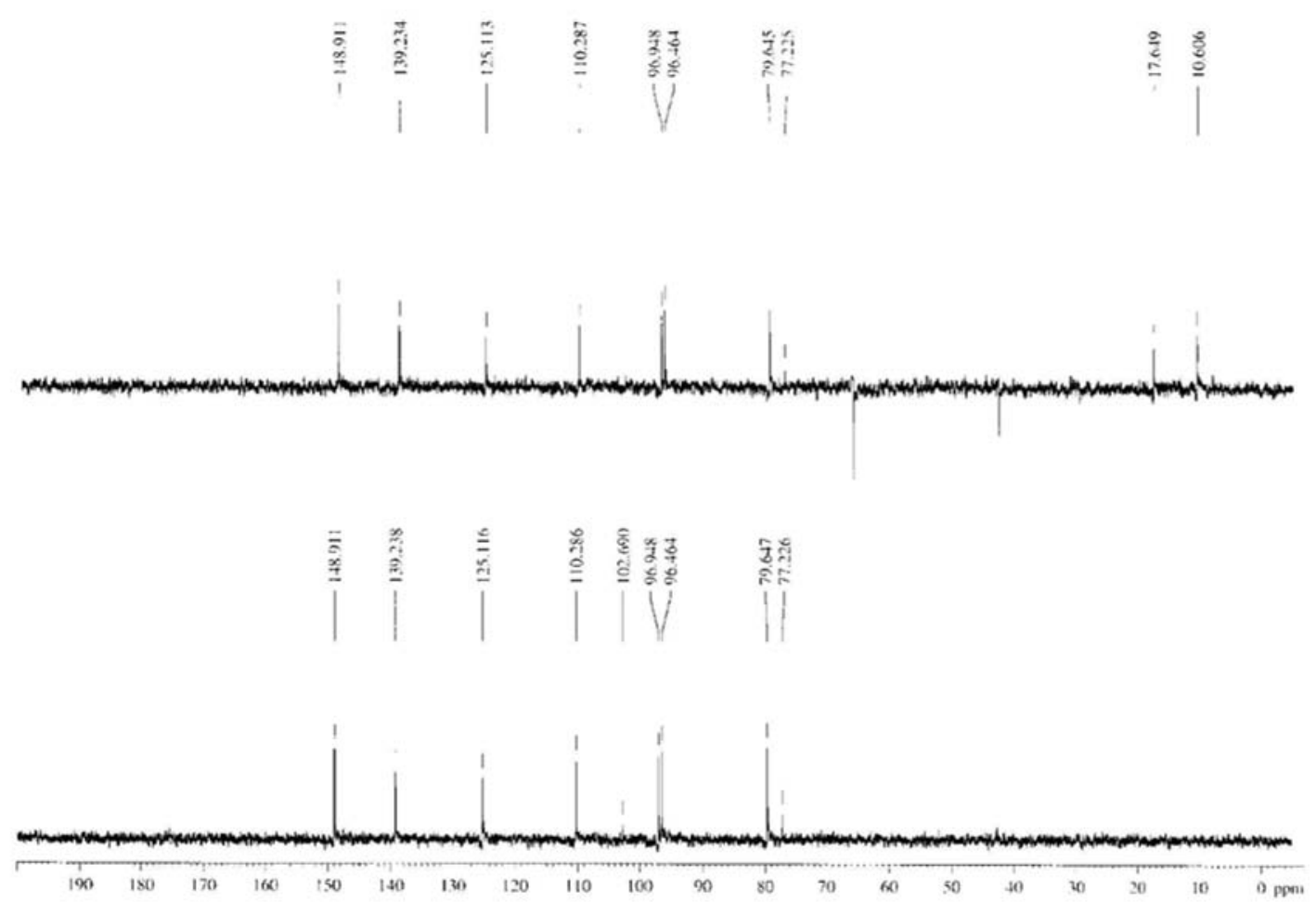

Figure S21. DEPT $135^{\circ}$ and $90^{\circ}$ spectrum of clausenalansimin B (9) $\left(100 \mathrm{MHz}, \mathrm{CDCl}_{3}\right)$. 
S12

New Coumarins from Clausena lansium Twigs

J. Braz. Chem. Soc.

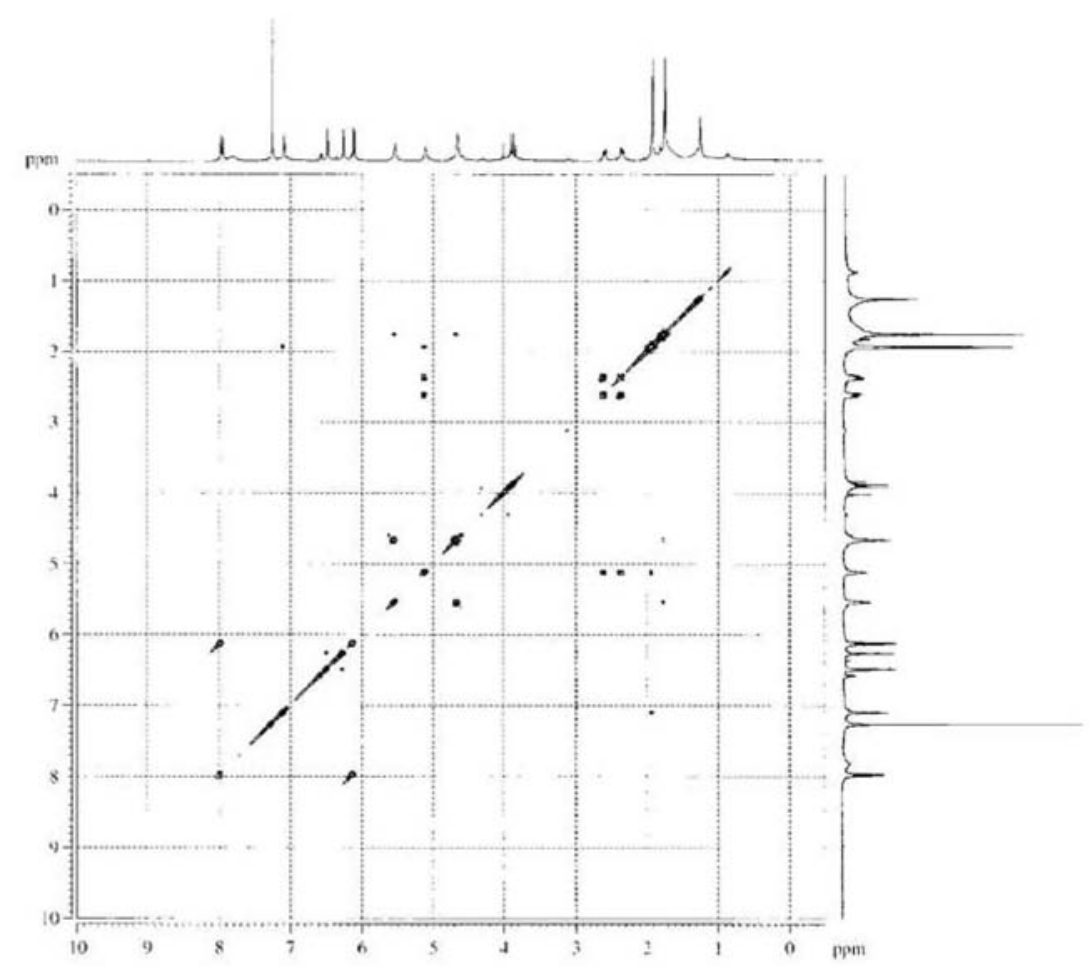

Figure S22. COSY spectrum of clausenalansimin B (9) (400 $\left.\mathrm{MHz}, \mathrm{CDCl}_{3}\right)$.

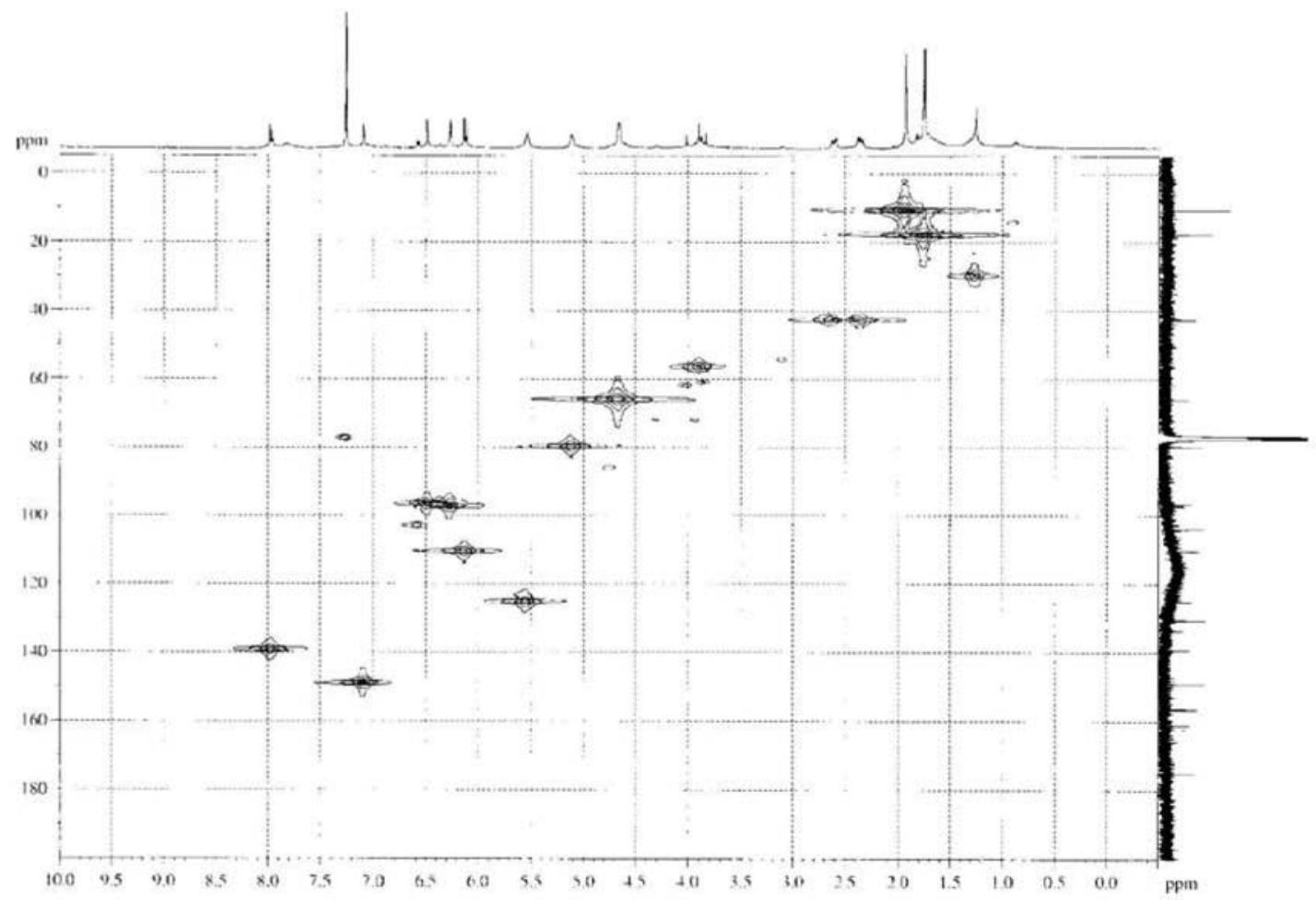

Figure S23. HMQC spectrum of clausenalansimin B (9) (400 MHz, $\left.\mathrm{CDCl}_{3}\right)$. 


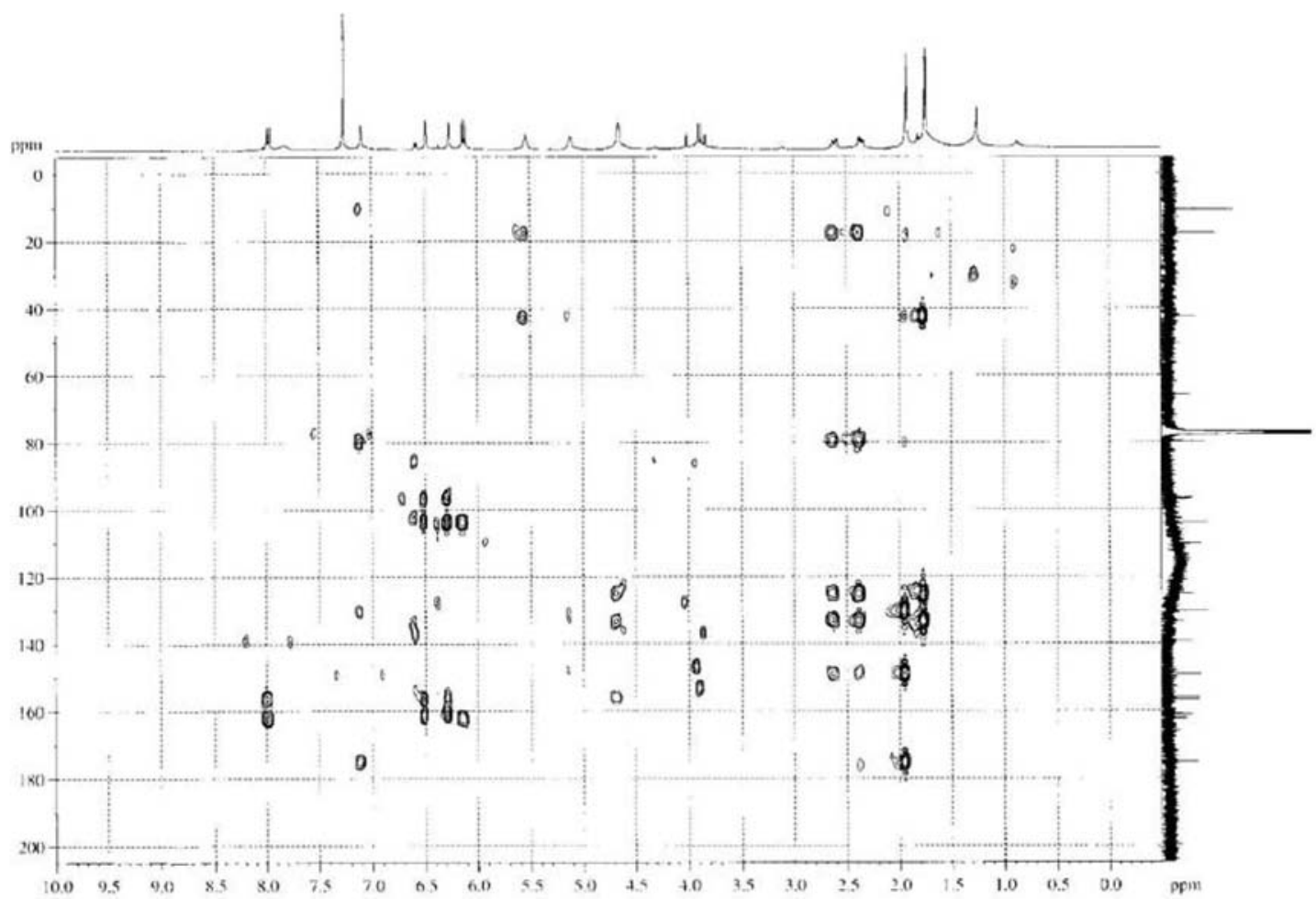

Figure S24. HMBC spectrum of clausenalansimin B (9) $\left(400 \mathrm{MHz}, \mathrm{CDCl}_{3}\right)$. 